\title{
Overview of Methods for Multi-Level and/or Multi-Disciplinary Optimization
}

\author{
A.J. de Wit*and F. van Keulen ${ }^{\dagger}$
}

\begin{abstract}
Multi-level optimization and multi-disciplinary optimization are areas of research that are concerned with developing efficient analysis and optimization techniques for complex systems that are made up of coupled elements (components). Within the field of multilevel optimization and multi-disciplinary optimization a large number of techniques have been developed for efficient analysis and optimization of complex systems. This paper presents an unified overview of main stream approaches that were found in the literature. Four general steps are distinguished in both multi-level optimization and multi-disciplinary optimization: physical coupling, optimization problem coupling, coordination and solution sequence. Via these four steps approaches are classified and possibilities for combining aspects of different methods are given. Finally, advantages and disadvantages of approaches applied to engineering problems are discussed and directions for further research are given. Keywords: multi-level, multi-disciplinary, optimization, decomposition, coordination, overview
\end{abstract}

\section{Introduction}

Complex systems, such as those encountered in aerospace engineering, can typically be considered a hierarchy of individual coupled elements. This hierarchy is reflected in the analysis techniques that are used to analyze the physical characteristics of the system. Consequently, a hierarchy of coupled models is to be used, accounting for different physical scales, components and/or disciplines. The total structural performance of such complex systems is a combination of responses evaluated at each level within the hierarchy. The design of complex systems with an embedded hierarchy via classical optimization approaches still involves adjusting a few parameters, often with respect to local objectives, not taking into account the influence such changes have on the overall design. Likewise, system-wide design objectives are not translated into element performance objectives and/or restrictions.

The field of multi-level optimization and multi-disciplinary optimization is concerned with developing efficient analysis and optimization techniques for complex systems that are made up of coupled elements (components). Multi-level optimization and multi-disciplinary optimization methods rely on a decomposition of the optimization problem into individual optimization problems that are coupled. Thus, it is attempted to incorporate design variables, objectives and constraints originating from different levels and/or disciplines into the design.

Over the last forty years a large number of multi-level optimization (e.g. Sobieszczanski-Sobieski et al. (1987), ${ }^{76}$ Alexandrov and Dennis (1994), ${ }^{1}$ Kim et al. (2003), ${ }^{41}$ amongst others) and multi-disciplinary optimization (e.g. Braun and Kroo (1997), ${ }^{22}$ Rodriguez et al. (2000), ${ }^{57}$ Sobieszczanski-Sobieski et al. (2000), ${ }^{71,72}$ amongst others) techniques have been developed. In this work a unified overview of main stream methods that were initially developed for either multi-level systems or multi-disciplinary systems is presented. This work focusses on the general steps of methods that belong to either the field of multi-level optimization or the field of multi-disciplinary optimization. Therefore, in this work no distinction is made between the two.

Previous literature overviews of either multi-level optimization or multi-disciplinary optimization distinguished between approaches on the basis of: single-level optimization versus multi-level optimization, ${ }^{13}$

${ }^{*}$ Collaborative Engineering Systems, National Aerospace Laboratory - NLR, Anthony Fokkerweg 2, 1059 CM Amsterdam, The Netherlands, e-mail: adewit@nlr.nl

${ }^{\dagger}$ Department of Precision and Microsystems Engineering, Delft University of Technology, Mekelweg 2, 2628 CD Delft, The Netherlands, e-mail: avankeulen@tudelft.nl 
positioning of computational costs and organization of the individual elements, ${ }^{74}$ positioning of consistency constraints, ${ }^{4}$ problem structure, ${ }^{82}$ amongst others. ${ }^{21,24,27,30,44-46,57,65,85}$ In this overview we focus on four generic steps: physical coupling, optimization problem coupling, coordination approach and solution sequence. These four generic steps enable us to classify each individual approach and show possibilities for combining aspects of different methods.

In this work a unified approach towards the multi-level and/or multi-disciplinary optimization of complex structures is presented. A unifying notation is introduced that treats all elements within the hierarchy equal. Two different decomposition approaches are identified and via an illustrative coupling circle a distinction between hierarchic and non-hierarchic decomposition is shown. Furthermore, via a problem matrix coupling within the optimization problem is made visible and four different optimization problems with embedded coupling are identified. Coordination techniques are introduced as part of the decision making process for which three different scenarios are presented. Numerical solution strategies are presented that depend on the coordination method chosen. A classification of multi-level and multi-disciplinary optimization methods is presented parallel to the steps taken to decompose and coordinate the optimization problem. Finally, advantages and disadvantages of approaches applied to engineering problems are discussed and directions for further research are given.

This extended abstract can be read as follows. In Section II a consistent notation is introduced that is used to identify general aspects of the multi-level and multi-disciplinary methods developed in the literature. Section III develops a generalized approach towards the decomposition of coupled elements. Coordination of the individual element solutions is discussed in Section [V] In addition, this section presents a classification of methods for multi-level and/or multi-disciplinary optimization methods found in the literature. Algorithm solution steps are discussed in Section V] Section VI]discusses some advantages and disadvantages of methods applied to engineering problems. In Section VII conclusions are presented.

\section{Unified Notation}

A multi-level problem typically has a hierarchical structure of individual elements as is illustrated in Figure 1(a). The top-level is denoted Level-0. At this level, the top-element or global design variables are distinguished, which are denoted ${ }^{0} \mathbf{x}$. One level lower, i.e. at Level-1, the "children" of Level-0 ("parent") can be found. The elements at Level-1 are numbered in the left upper corner, i.e. "x. At the second level, Level-2,

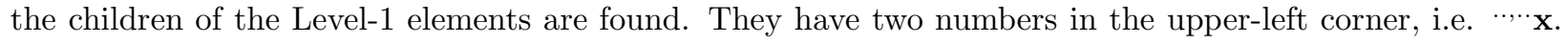
The first reflects the parent in the hierarchy and the second their place among the children of the parent element. In a similar fashion, more levels can be added to the hierarchy. Consequently, at every level the relative position can easily be seen from the superscript in front of a (design) variable. Similarly, the physical responses corresponding to the elements are identified. This is illustrated in Figure 1(b).

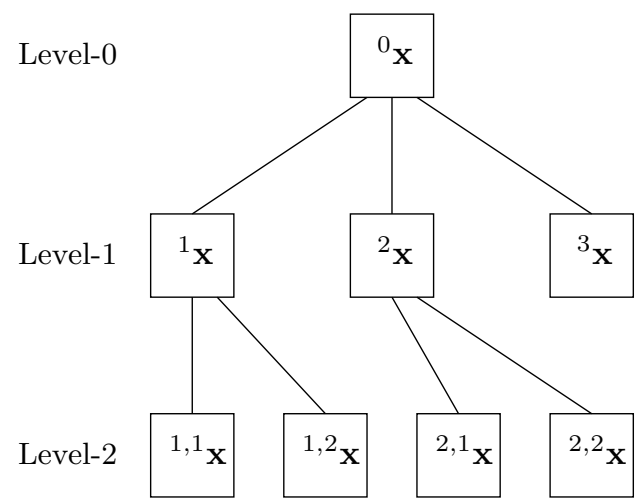

(a)

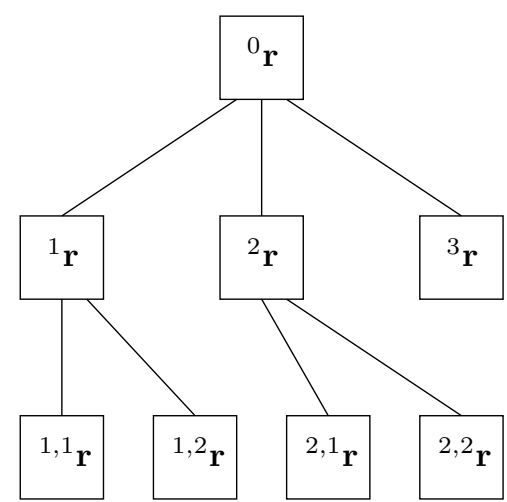

(b)

Figure 1. Multi-level notation for a three-level hierarchy. (a) The design variables corresponding to the individual elements are illustrated. (b) The physical responses corresponding to each of the elements.

Although for each element a set of physical responses can be identified, these responses are typically interacting with responses in neighboring elements, i.e. parent, brothers/sisters and children. In other words, there are couplings which need to be taken into account as is illustrated in Figure 2, For this purpose, the operators $\mathcal{H}$ are introduced. These operators map the response from one element, onto another. In front 
Level-0

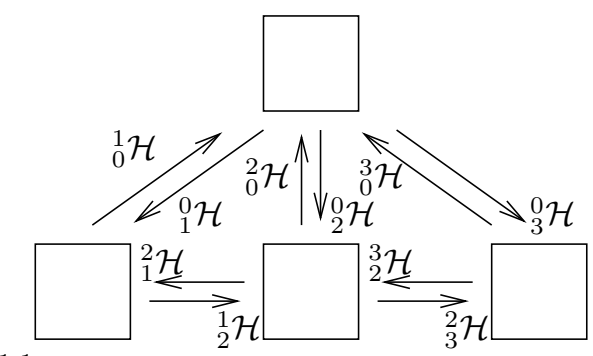

Level-1

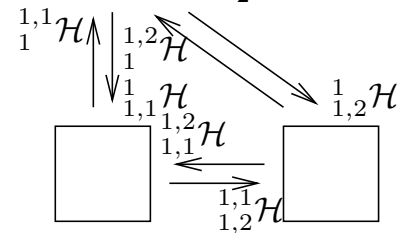

Level-2

1,2 H

Figure 2. Physical interactions between elements. The operators $: \cdot \mathcal{H}$ map the physical responses of an element onto a neighboring element. This can be a mapping taking place at the same level, though it may also map between different levels.

Level-0

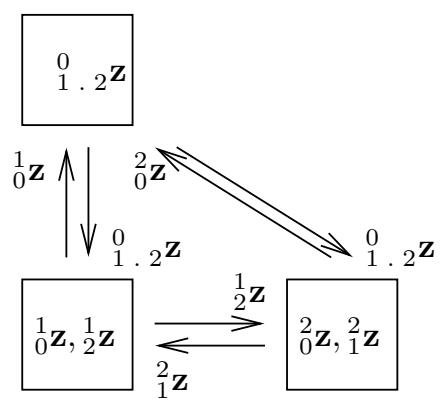

Figure 3. Design variables that are shared among multiple elements. The top left index represents the element to which the design variable is associated and the bottom index represents the neighboring element(s) that share the design variable.

of the operator in the top-left corner the origin of the information is indicated, i.e. ${ }^{\cdots} \mathcal{H}$. In the bottom-left corner, the destination of the information is specified, i.e. .. $\mathcal{H}$.

Design variables can be present that are shared among multiple elements. These design variables are considered separately from the individual design variables $\cdot \mathbf{x}$. The shared design variables are written as.. $\mathbf{z}$. The top-left index (*) represents the element to which the design variable is assigned and the bottom-left index (..) represents the element that shares that design variable. In case the same design variable is shared among the parent element and two or more child elements a $\operatorname{dot}(\ldots$ z $)$ separates the indices from each of the child elements. Shared design variables are shown in Figure 3, where a design variable is shared among three elements distributed over two levels of a hierarchy.

Responses that are mapped from one element onto another element are introduced via the operator $\mathcal{H}$. Once a response is mapped from one element onto another element it is written as :..h $\mathbf{h}$, see Figure 4 This variable is called a coupling variable and is defined as: $\cdot \mathbf{h}=\cdot \cdot \mathcal{H}(\cdot \cdot \mathbf{r})$. This coupling variable can be used in one element as a desirable property of neighboring elements. Hence, instead of mapping the physical responses coming from a neighboring element onto the current element: $: \because \mathcal{H}(\cdot \mathbf{r})=\cdot \cdot \mathbf{h}$, the value of the coupling variable $(\because \mathbf{n})$ could be chosen. The corresponding physical responses $(\cdot \mathbf{r})$ are determined by the neighboring element.

Consistency constraints are introduced in order to temporarily decouple the elements from their surroundings, such that each of them is solved without interacting with other elements. These consistency constraints are mathematically defined as: $: . \mathbf{c}=\cdot . \cdot \mathcal{H}(\cdot \cdot \mathbf{r})-\cdot . \cdot \mathbf{h}$ and ${ }_{j}^{i} \mathbf{c}_{z}={ }_{j}^{i} \mathbf{z}-{ }_{i}^{j} \mathbf{z}(i \neq j)$. A consistency constraint that is assigned to an element enables the element to change ${ }^{*} \mathbf{r}$ and/or ${ }_{j}^{i} \mathbf{z}$ or when applicable ${ }^{*} \mathbf{h}$ and/or ${ }_{i}^{j} \mathbf{z}$ without communicating directly to the element to which it is coupled. These consistency constraints are written.. c and $: . \mathbf{c}_{z}$, where the upper left subscript ${ }^{\prime} \cdot \mathbf{c}, \cdot{ }^{\prime} \mathbf{c}_{z}$ indicates the origin of the mapped responses and/or shared design variables and the lower left subscript ..., ... $\mathbf{c}_{z}$ indicates the destination, see Figure 4. This notation is in accordance with the notation for coupling (hence operators $\mathcal{H}$ ) or shared design variables $(\mathbf{z})$ between 
two individual elements. Decoupled hierarchical elements depend on consistency constraints, therefore the consistency constraints are added to the optimization functions inside the hierarchical elements.

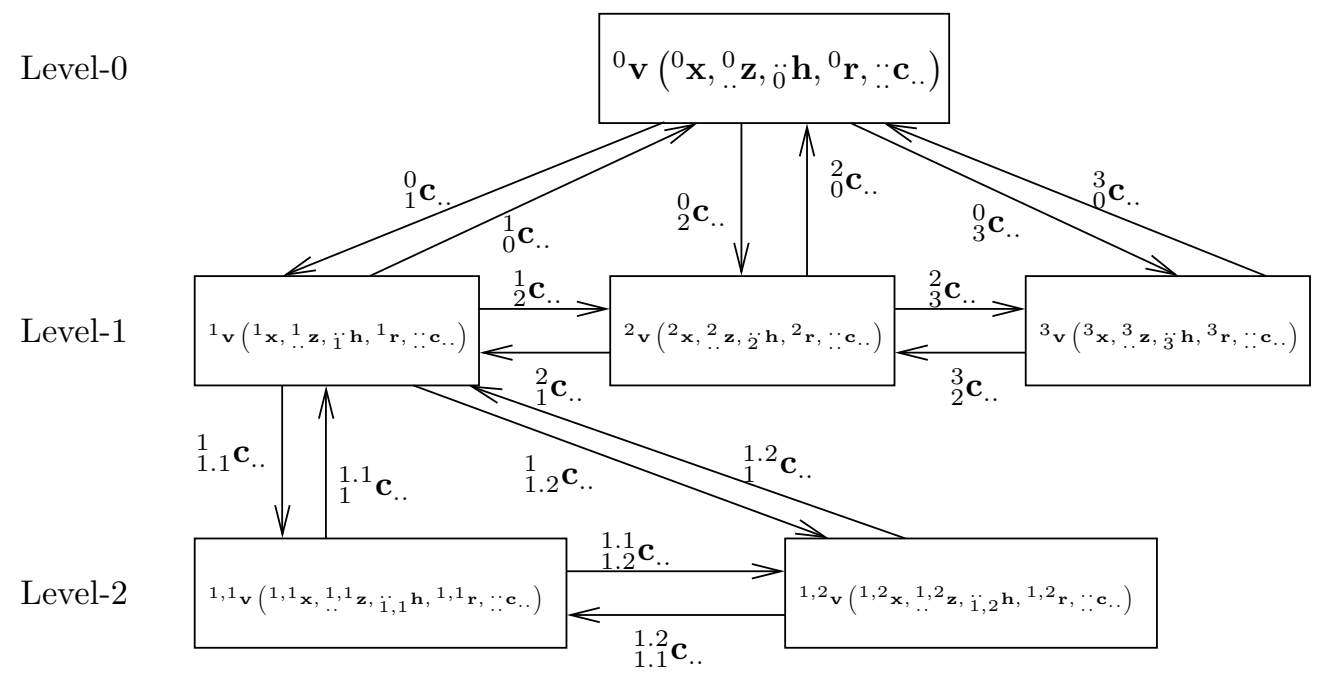

Figure 4. Functions used in the optimization formulation of the hierarchy. These functions typically depend on design variables, the constraints and the physical responses of the corresponding element.

The final step in setting up an optimization problem is to formulate objective and constraint functions $(\cdot \mathbf{v})$ that can be minimized (maximized) by the optimizer. These functions depend on local design variables $(\cdot \mathbf{x})$, local responses $(\cdot \mathbf{r})$ and consistency conditions $(\cdot \mathbf{c})$ as well as the coupling $(\cdot \cdot \mathbf{h}$ and $\cdot \mathbf{z})$ between two individual elements. These design functions are illustrated in Figure 4.

\section{Decomposition}

Decomposition involves identifying (weak) links between elements that are coupled, allowing the elements to represent individual, though coupled, optimization problems. The origin of this coupling can be physically oriented or can be prompted by the nature of the design optimization problem at hand. Generic properties of physical coupling between elements is captured via an illustrative coupling circle (Section III.A) and the coupling within the optimization problem is shown via a problem matrix (Section III.B).

\section{III.A. Physical coupling}

Physical coupling between two elements is shown in Figure 5(a). A top element (Level-0) produces a physical response ${ }^{0} \mathbf{r}$ which is mapped into information ${ }_{1}^{0} \mathbf{h}$ that is necessary to compute the physical responses at the bottom (Level-1) element. The bottom element computes a physical response which is mapped back to the top element.

Decoupling is accomplished via consistency constraints. There are two formulations that are used frequently in order to maintain consistency between two coupled elements. These formulations are shown in Figure 5(b) and Figure 5(c) and can mathematically be expressed as:

1. strong (equality) constraints/conditions:

$$
\begin{aligned}
& { }_{1}^{0} \mathbf{c}={ }_{1}^{0} \mathcal{H}\left({ }^{0} \mathbf{r}\right)-{ }_{1}^{0} \mathbf{h}=\mathbf{0}, \\
& { }_{0}^{1} \mathbf{c}={ }_{0}^{1} \mathcal{H}\left({ }^{1} \mathbf{r}\right)-{ }_{0}^{1} \mathbf{h}=\mathbf{0} .
\end{aligned}
$$

2. weak (relaxation), e.g. using Lagrange multipliers:

$$
\begin{aligned}
& { }_{1}^{0} \boldsymbol{\lambda}^{T 0} \mathbf{c}={ }_{1}^{0} \boldsymbol{\lambda}^{T}\left({ }_{1}^{0} \mathcal{H}\left({ }^{0} \mathbf{r}\right)-{ }_{1}^{0} \mathbf{h}\right), \\
& { }_{0}^{1} \boldsymbol{\lambda}^{T}{ }_{0}^{1} \mathbf{c}={ }_{0}^{1} \boldsymbol{\lambda}^{T}\left({ }_{0}^{1} \mathcal{H}\left({ }^{1} \mathbf{r}\right)-{ }_{0}^{1} \mathbf{h}\right) .
\end{aligned}
$$

Other common relaxation formulations involve penalty functions ${ }^{39}$ or an augmented Lagrangian ${ }^{10}$ function. Each decomposition formulation can be subdivided into:

1. hierarchic decomposition, which is subdivided into top-down or/and bottom-up formulations. 


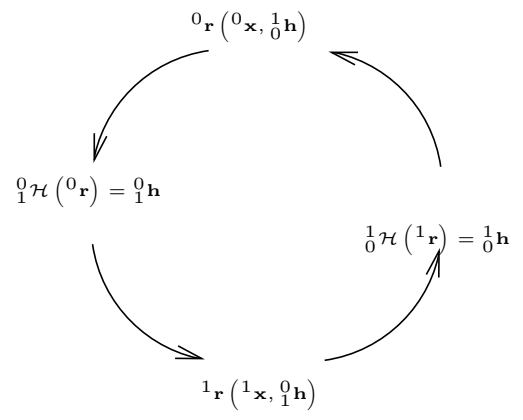

(a)
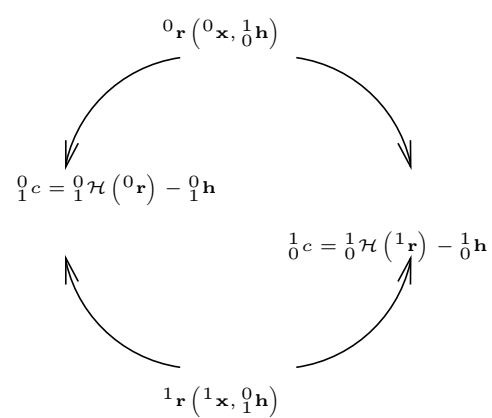

(b)
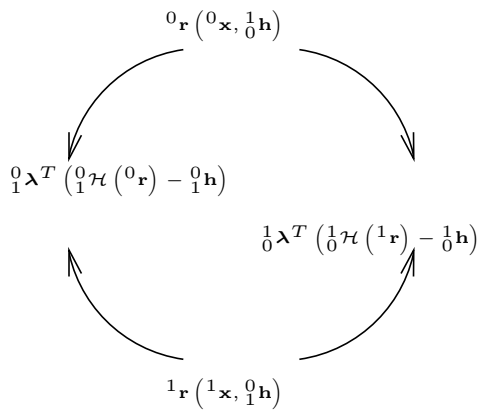

(c)

Figure 5. (a) Physical response interaction between two elements. The responses ${ }^{0} \mathrm{r}$ computed at the parent element are transformed by the operator ${ }_{1}^{0} \mathcal{H}$ into information ${ }_{1}^{0} \mathrm{~h}$ influencing the responses of the child element ${ }^{1} \mathrm{r}$. Likewise, the responses from the child element are transformed by the operator ${ }_{0}^{1} \mathcal{H}$ into information ${ }_{0}^{1} h$ influencing the responses of the parent element. (b) Consistency between the coupled elements is maintained through interface compatibility or equilibrium. (c) Consistency is relaxed using Lagrange multipliers. Typically, these multipliers are controlled, such that the inconsistencies vanish at compatibility or equilibrium of the interface.

2. non-hierarchic decomposition, which treats all elements equal.

Hierarchic decomposition is the result of identifying elements in the hierarchy that dictate the output from other elements. This is shown in Figure 6(a) in case of a top-down decomposition with equality consistency constraints. In this figure, the top Level-0 element prescribes the necessary output from the Level-1 element. Consistency constraints are assigned to the bottom element constraining the right part of the coupling circle. A similar but opposite approach is possible, where the Level-1 element prescribes the necessary output of the Level-0 element. The latter approach is shown in Figure 6(b) and is called a bottom-up decomposition constraining the left part of the coupling circle.

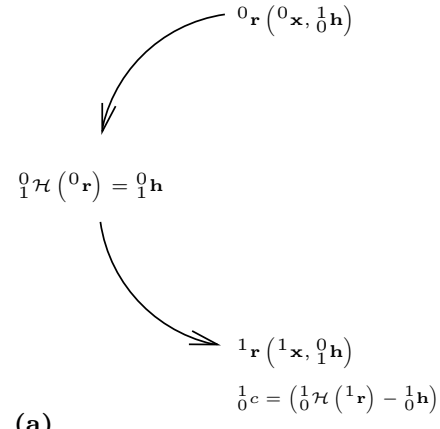

(a)

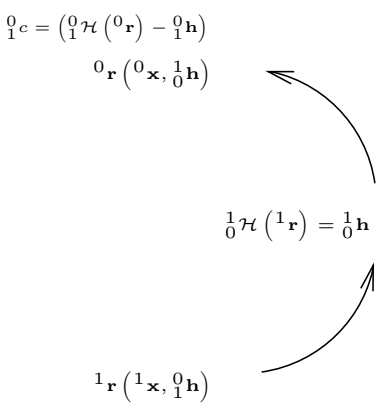

(b)
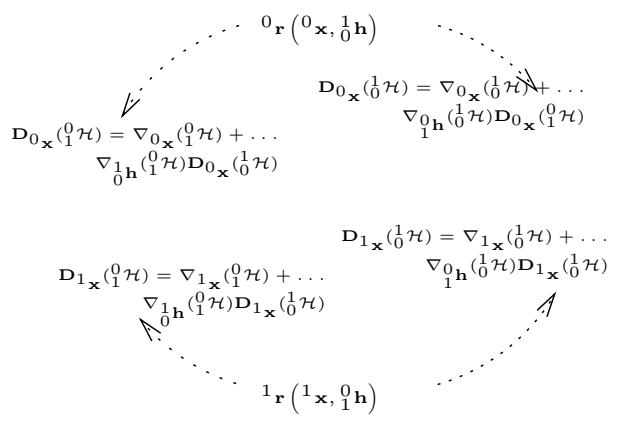

(c)

Figure 6. (a) Top-down decomposition via consistency constraints formulated as an equality. The Level-0 element prescribes the necessary responses for the Level-1 element via the equality consistency constraints. (b) Bottom-up decomposition via consistency constraints formulated as an equality. The Level-1 element prescribes the necessary responses for the Level-0 element via the equality consistency constraints. (c) Non-hierarchic decomposition, none of the elements prescribes the output of a neighboring element. Equality consistency constraints are assumed $(. . . \mathrm{c}=0)$ and the interaction is approximated via sensitivity calculations (Global Sensitivity Equations).

Non-hierarchic decomposition involves elements that do not prescribe output from each other. Instead, output is estimated and after element solutions are found information is exchanged among the elements to update the input parameters via a coordination method.

In case decomposition is accomplished via equality constraints the non-hierarchic decomposition is shown in Figure 6(c). Additional information is required to take into account changes in the interaction. This is accomplished via derivation of the Global Sensitivity Equations. ${ }^{62,66}$ The Global Sensitivity Equations for the two coupled elements considered are found via applying the chain rule:

$$
\begin{aligned}
& \mathbf{D}_{0_{\mathbf{x}}}\left({ }_{1}^{0} \mathcal{H}\right)=\nabla_{\mathbf{o}_{\mathbf{x}}}\left({ }_{1}^{0} \mathcal{H}\right)+\nabla_{1}^{1} \mathbf{h}\left({ }_{1}^{0} \mathcal{H}\right) \mathbf{D}_{0_{\mathbf{x}}}\left({ }_{0}^{1} \mathcal{H}\right) \\
& \mathbf{D}_{\mathbf{1}_{\mathbf{x}}}\left({ }_{1}^{0} \mathcal{H}\right)=\nabla_{\mathbf{1}_{\mathbf{x}}}\left({ }_{1}^{0} \mathcal{H}\right)+\nabla_{0} \mathbf{h}\left({ }_{1}^{0} \mathcal{H}\right) \mathbf{D}_{1_{\mathbf{x}}}\left({ }_{0}^{1} \mathcal{H}\right) \\
& \mathbf{D}_{0_{\mathbf{x}}}\left({ }_{0}^{1} \mathcal{H}\right)=\nabla_{\mathbf{o}_{\mathbf{x}}}\left({ }_{0}^{1} \mathcal{H}\right)+\nabla_{0} \mathbf{h}\left({ }_{0}^{1} \mathcal{H}\right) \mathbf{D}_{0_{\mathbf{x}}}\left({ }_{1}^{0} \mathcal{H}\right) \\
& \mathbf{D}_{\mathbf{1}_{\mathbf{x}}}\left({ }_{0}^{1} \mathcal{H}\right)=\nabla_{\mathbf{1}_{\mathbf{x}}}\left({ }_{0}^{1} \mathcal{H}\right)+\nabla_{0} \mathbf{h}\left({ }_{0}^{1} \mathcal{H}\right) \mathbf{D}_{1_{\mathbf{x}}}\left({ }_{1}^{0} \mathcal{H}\right)
\end{aligned}
$$




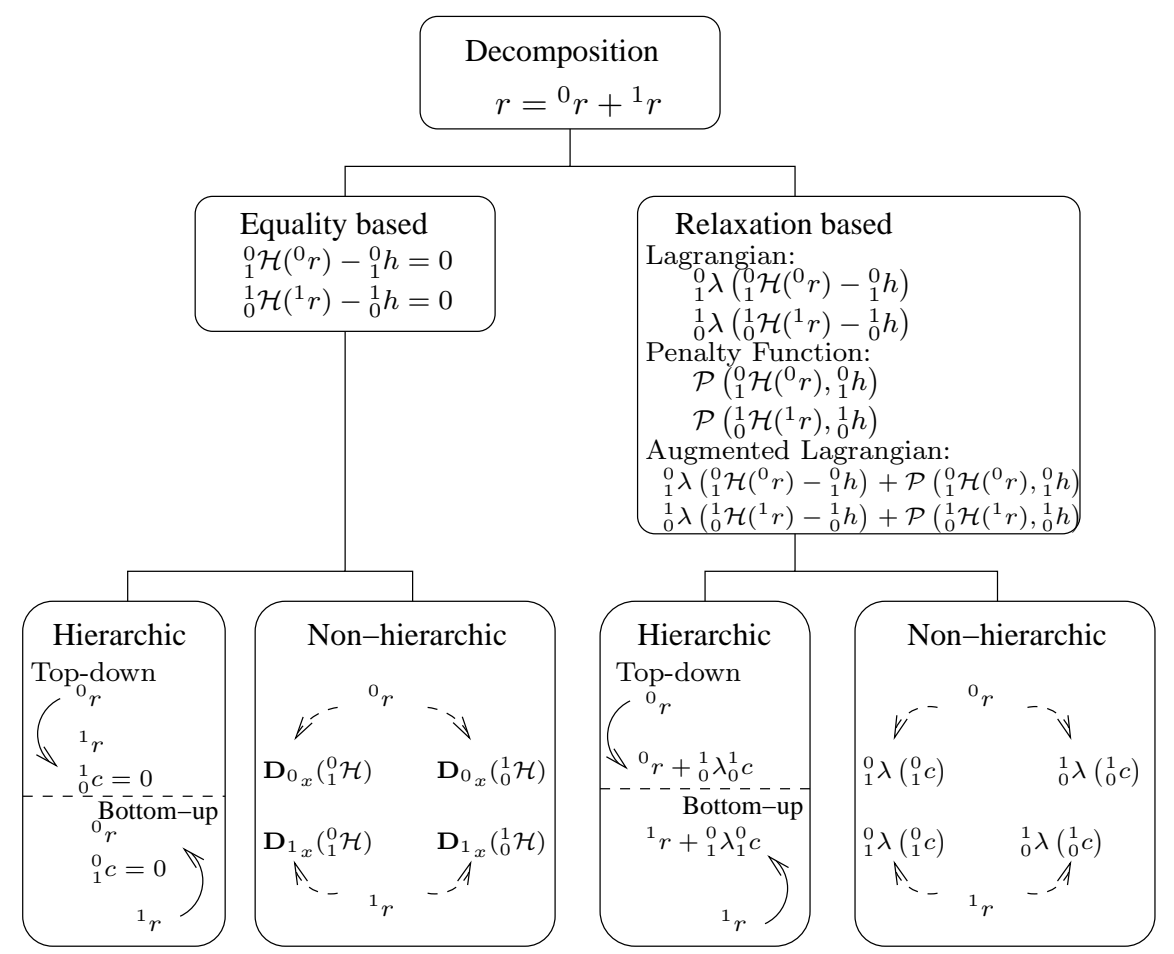

Figure 7. Summary of the decomposition process. Two choices are available for decomposing coupled problems. These are equality based decomposition and relaxation based decomposition. Furthermore, these approaches are subdivided in hierarchic top-down or bottom-up formulations and non-hierarchic formulations.

where $\nabla_{\mathbf{x} . .}=\frac{\partial . .}{\partial x_{1}}+\ldots+\frac{\partial . .}{\partial x_{n}}$ and the total derivatives are defined as $\mathbf{D}_{\mathbf{h} . .}=\left[\frac{\mathrm{d} . . .}{\mathrm{d} h_{1}}, \ldots, \frac{\mathrm{d} . .}{\mathrm{d} h_{n}}\right]^{T}$.

The necessary sensitivity information is the solution to the system of equations:

$$
\begin{aligned}
& {\left[\begin{array}{cc}
\mathbf{I} & -\nabla_{0} \mathbf{h}\left({ }_{1}^{0} \mathcal{H}\right) \\
-\nabla_{0} \mathbf{h}\left({ }_{0}^{1} \mathcal{H}\right) & \mathbf{I}
\end{array}\right]\left[\begin{array}{ll}
\mathbf{D}_{\mathbf{x}_{\mathbf{x}}}\left({ }_{1}^{0} \mathcal{H}\right) & \mathbf{D}_{\mathbf{1}_{\mathbf{x}}}\left({ }_{1}^{0} \mathcal{H}\right) \\
\mathbf{D}_{\mathbf{x}_{\mathbf{x}}}\left({ }_{0}^{1} \mathcal{H}\right) & \mathbf{D}_{\mathbf{1}_{\mathbf{x}}}\left({ }_{0}^{1} \mathcal{H}\right)
\end{array}\right]=\ldots} \\
& {\left[\begin{array}{cc}
\nabla_{\mathbf{o}_{\mathbf{x}}}\left({ }_{1}^{0} \mathcal{H}\right) & \mathbf{0} \\
\mathbf{0} & \nabla_{1_{\mathbf{x}}}\left({ }_{0}^{1} \mathcal{H}\right)
\end{array}\right]}
\end{aligned}
$$

An additional technique is required to monitor consistency between the individual elements after each individual element is evaluated.

Figure 7 presents an overview of the two-decomposition techniques for coupled elements: equality based decomposition; and relaxation based decomposition. Relaxation based decomposition is accomplished via relaxation of the consistency constraints. There are three typical approaches that relax these constraints, e.g. Lagrangian relaxation, Penalty function relaxation and Augmented Lagrangian relaxation. Both decomposition techniques can be subdivided into hierarchic and non-hierarchic formulations.

Up to this point, the discussion has focused on physical coupling between elements of a hierarchy. This coupling is embedded in the optimization problem and can be made visible via, e.g., the problem matrix. This problem matrix is discussed in the next section.

\section{III.B. Problem matrix}

The decomposition process of optimization problems involves identifying relationships between the design variables, physical responses and objectives/constraints that permit us to separate them into individual elements that are connected. These optimization problems for which no elements have been identified yet 
are defined as:

$$
\begin{array}{cl}
\min _{\mathbf{x}} & v_{f}(\mathbf{x}, \mathbf{r}(\mathbf{x})) \\
\text { s.t. } & \mathbf{v}_{g}(\mathbf{x}, \mathbf{r}(\mathbf{x})) \leq \mathbf{0} \\
& \mathbf{v}_{h}(\mathbf{x}, \mathbf{r}(\mathbf{x}))=\mathbf{0}
\end{array}
$$

where $v_{f}(\ldots)$ is the objective function, $\mathbf{v}_{g}(\ldots)$ are inequality constraints and $\mathbf{v}_{h}(\ldots)$ are equality constraints. The relationship between variables $(\mathbf{x})$, responses $(\mathbf{r})$ and functions $\left(v_{f}, \mathbf{v}_{g}\right.$ and $\left.\mathbf{v}_{h}\right)$ is illustrated, amongst others by Barthelemy, ${ }^{15}$ with the problem matrix (also known as Functional Dependence Table ${ }^{85}$ ).

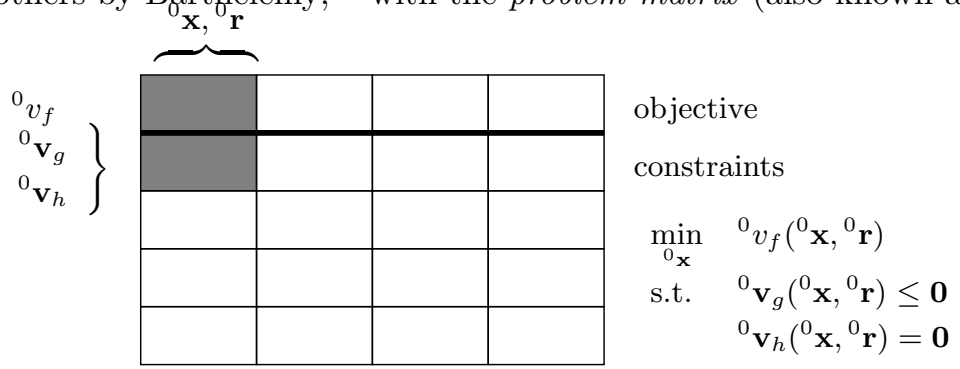

Figure 8. Problem matrix, illustrating the relationship between variables and functions. A dark colored block indicates that the functions (horizontally) depend on the design variables and responses (vertically).

A single individual element depends on local design variables and local physical responses. Such an element is illustrated in Figure 8, where the dependence of $\mathbf{r}$ on the design variables $\mathbf{x}$ is dropped for brevity of notations. Above the thick black line the objective function ${ }^{0} v_{f}$ is written and the block of variables ${ }^{0} \mathbf{x}$ and responses ${ }^{0} \mathbf{r}$ that this objective function depends on is dark. Likewise, the design constraints ${ }^{0} \mathbf{v}_{g}$ (inequality) and ${ }^{0} \mathbf{v}_{h}$ (equality) are listed below the thick black line and the block of variables and responses corresponding to these functions is colored dark.

For a problem consisting of many elements, the simplest case is when there are only uncoupled problems. ${ }^{43}$ The problem matrix resembles that of Figure 9. A hierarchy of three individual elements is shown that are uncoupled. The dependencies of optimization functions (horizontal) on design variables and physical responses (vertical) form a diagonal pattern in the problem matrix. This is called a block diagonal problem matrix. The constraints are completely separable and the objective function is additively separable, meaning that the objective function consists of a summation of element objectives that are uncoupled. Combined these element objectives form the entire objective function. The element objectives are minimized separately. Uncoupled problems can be solved independently and therefore there is no coordination (see Section IV necessary.

In order to make the problem more manageable, one searches for interaction (or coupling) between groups of variables and/or responses such that individual elements are formed or identified. These interactions are subdivided into:

1. non existing, hence elements that are formed or identified are uncoupled.

2. weak, if the number of coupling variables is substantially less than the total number of variables associated with each of the individual elements and none of the coupling variables that are identified has a significant impact on the solution of the individual element.

3. strong, if most of the coupling variables are shared among the elements and a diagonal on the problem matrix cannot be identified.

In real life problems, uncoupled elements do not exist. But often a distinction between weak and strong dependencies of the elements can be made. In Figure 10 such a distinction is illustrated. The light gray blocks indicate weak dependencies and the dark gray blocks strong dependencies.

Preferably one has a Block Diagonal problem matrix such as illustrated in Figure 9 since the individual elements are then uncoupled in the constraints and additively separable in the objective function. However, more frequently encountered problems involve coupling. In that case, the subproblems are connected through design ${ }_{j}^{i} \mathbf{z}$ and/or coupling variables ${ }_{j}^{i} \mathbf{h}$. Shared design variables ${ }_{j}^{i} \mathbf{z}$ are design variables that are present in multiple hierarchical elements. Coupling variables ${ }_{j}^{i} \mathbf{h}$ represent the mapped physical responses $\left({ }_{j}^{i} \mathcal{H}\left({ }^{i} \mathbf{r}\right)\right)$ from 


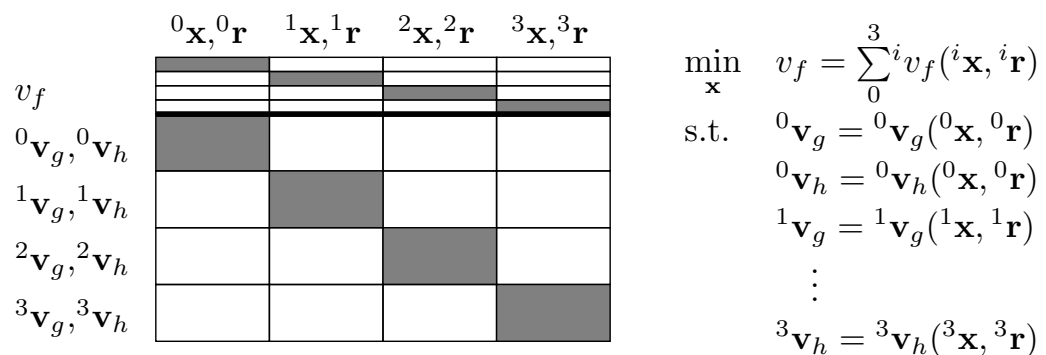

Figure 9. Block diagonal matrix, showing the relationships between variables and fully uncoupled functions. In this case there is no need for coordinating the solution process to reach the optimum of the multi-level optimization problem.

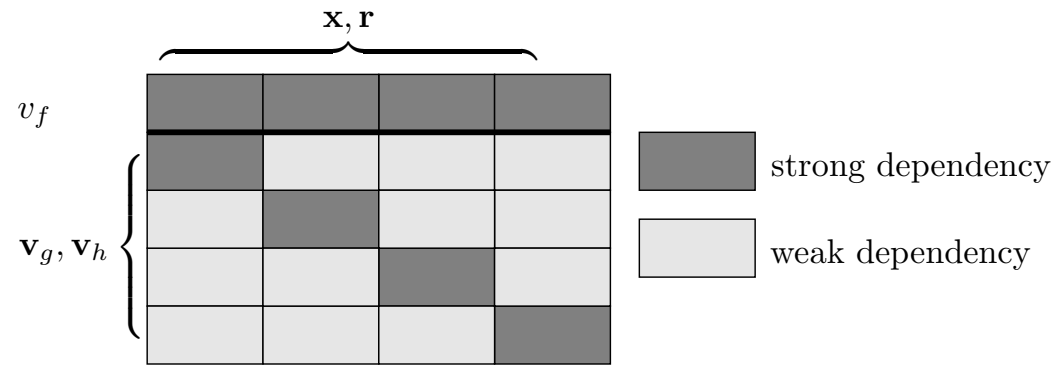

Figure 10. Problem matrix, illustrating the weak (off-diagonal) and strong (main-diagonal) dependencies between individual elements. The objective is a function of all the design variables and depends on all the elements. However, the constraints can be organized in strong and weak dependencies associated with each individual element.

one element onto a neighboring element to which it is coupled. An example of a problem consisting of shared design variables and coupled responses is the minimization of an additively separable objective function subjected to constraints:

$$
\begin{aligned}
& \min _{\mathbf{x}} \quad{ }^{0} v_{f}\left({ }_{i}^{0} \mathbf{z},{ }^{0} \mathbf{x},{ }^{0} \mathbf{r}\left({ }_{i}^{0} \mathbf{z},{ }^{0} \mathbf{x},{ }_{0}^{i} \mathbf{h}\right)\right)+\sum_{i=1}^{3}{ }^{i} v_{f}\left({ }_{0}^{i} \mathbf{z},{ }^{i} \mathbf{x},{ }^{i} \mathbf{r}\left({ }_{0}^{i} \mathbf{z},{ }^{i} \mathbf{x},{ }_{i}^{0} \mathbf{h}\right)\right) \\
& \text { s.t. } \quad{ }^{0} \mathbf{v}_{g}\left({ }_{i}^{0} \mathbf{z},{ }^{0} \mathbf{x},{ }^{0} \mathbf{r}\left({ }_{i}^{0} \mathbf{z},{ }^{0} \mathbf{x},{ }_{0}^{i} \mathbf{h}\right)\right) \leq \mathbf{0} \\
& { }^{i} \mathbf{v}_{g}\left({ }_{0}^{i} \mathbf{z},{ }^{i} \mathbf{x},{ }^{i} \mathbf{r}\left({ }_{0}^{i} \mathbf{z},{ }^{i} \mathbf{x},{ }_{i}^{0} \mathbf{h}\right)\right) \leq \mathbf{0} \quad i=1, \ldots, 3 \\
& { }^{0} \mathbf{v}_{h}\left({ }_{i}^{0} \mathbf{z},{ }^{0} \mathbf{x},{ }^{0} \mathbf{r}\left({ }_{i}^{0} \mathbf{z},{ }^{0} \mathbf{x},{ }_{0}^{i} \mathbf{h}\right)\right)=\mathbf{0} \\
& { }^{i} \mathbf{v}_{h}\left({ }_{0}^{i} \mathbf{z},{ }^{i} \mathbf{x},{ }^{i} \mathbf{r}\left({ }_{0}^{i} \mathbf{z},{ }^{i} \mathbf{x},{ }_{i}^{0} \mathbf{h}\right)\right)=\mathbf{0} \\
& \text { where } \quad{ }^{0} \mathbf{x},{ }^{0} \mathbf{r} \quad \text { top-element level variables } \\
& { }^{i} \mathbf{x},{ }^{i} \mathbf{r} \quad \text { element level variables } \\
& \cdot \mathbf{h} \quad \text { coupling variables } \\
& \cdots \mathbf{z} \text { shared design variables } \\
& \mathbf{x}=\left[{ }^{0} \mathbf{x},{ }_{i}^{0} \mathbf{z},{ }^{i} \mathbf{x},{ }_{0}^{i} \mathbf{z}\right] \\
& { }_{i}^{0} \mathcal{H}\left({ }^{0} \mathbf{r}\right)={ }_{i}^{0} \mathbf{h} \\
& { }_{0}^{i} \mathcal{H}\left({ }^{i} \mathbf{r}\right)={ }_{0}^{i} \mathbf{h} \\
& { }_{i}^{0} \mathbf{z}={ }_{0}^{i} \mathbf{z}
\end{aligned}
$$

Notice that global variables ${ }^{0} \mathbf{x}$ and ${ }^{0} \mathbf{r}$ related to the Level-0 problem, local variables ${ }^{i} \mathbf{x}$ and ${ }^{i} \mathbf{r}$, which are related to Level-1, coupling variables ...h connecting the two levels via physical coupling and shared design variables that are shared among individual elements $\left({ }_{j}^{i} \mathbf{z}={ }_{i}^{j} \mathbf{z}\right)$ are distinguishable. The current example involves four individual elements that are coupled and divided over two levels. The problem matrix that illustrates the relations between the variables of Equation 4 is called an Angular problem matrix. This problem matrix is illustrated in Figure 11 for the problem of Equation 4.

Equation 4 represents an optimization problem where coupling is present. No decomposition technique is introduced yet, however a hierarchy within the problem is made visible via the multi-level notation. To show how decomposition enters the optimization problem formulation a hierarchic top-down decomposition is applied. The coupling circle shown in Figure 12 (a) shows the physical coupling that is present between the two levels. The physical coupling is decomposed and consistency is maintained between Level-0 and Level-1 elements through consistency constraints, e.g., top-down hierarchic decomposition shown in Figure 12(b). 


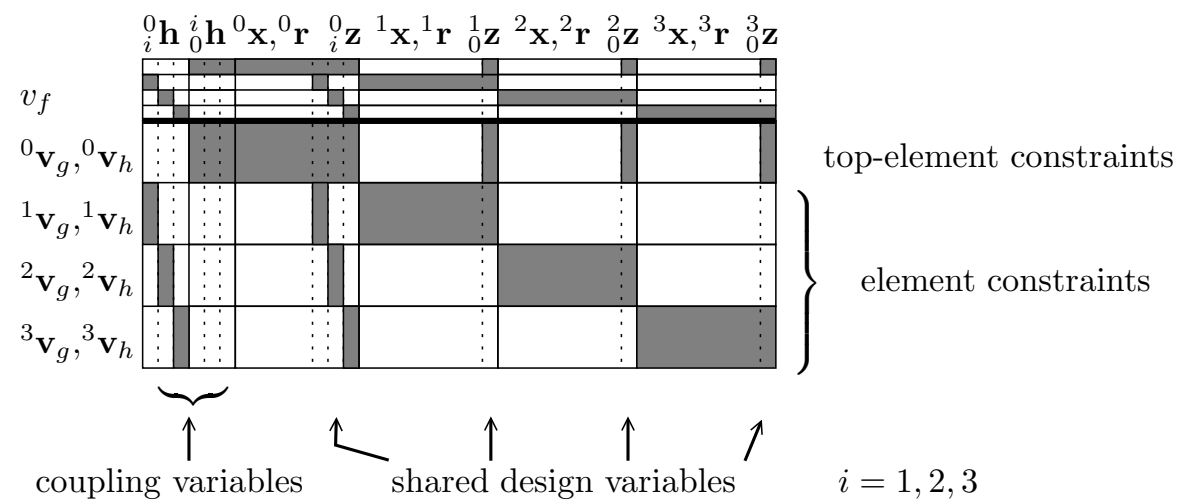

Figure 11. Angular problem matrix, coordination between levels is necessary due to the coupling variables ${ }_{i}^{0} \mathrm{~h}$ and ${ }_{0}^{i} \mathrm{~h}$. Each element receives a copy of the variable ${ }_{0}^{i} \mathrm{~h}$ or ${ }_{i}^{0} \mathrm{~h}$ and coordination is necessary to introduce these copies into the other element.

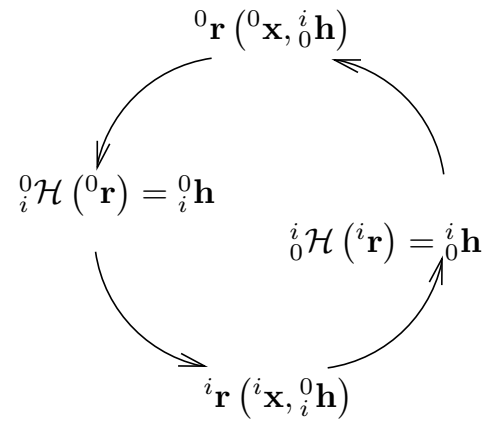

(a)

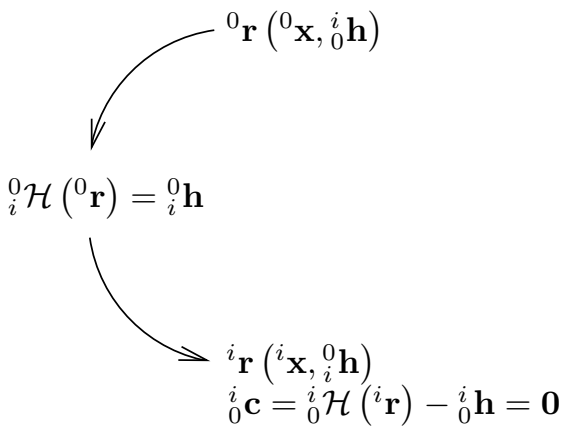

(b)

Figure 12. (a) Physical response interaction between a single Level-0 element and the $i^{\text {th }}$ element present at Level-1, where $i=1, \ldots, n \mathbf{n}$ being the amount of elements present at Level-1. The responses ${ }^{0} \mathbf{r}$ computed at the parent element are transformed by the operator ${ }_{i}^{0} \mathcal{H}$ into information that influences the responses of the child element ${ }^{i} \mathbf{r}$. Likewise, the responses from the child element are transformed by the operator ${ }_{0}^{i} \mathcal{H}$ into information that influences the responses at the parent element. (b) The Level-0 element prescribes the necessary responses for the Level-1 element by means of equality consistency constraints. This is called top-down decomposition.

Due to the top-down hierarchic decomposition, Level-0 does no longer receive the coupling variables ${ }_{0}^{i} \mathbf{h}$ from Level-1. Instead, values for ${ }_{0}^{i} \mathbf{h}$ are estimated and used during Level-0 computations. Since the responses from Level-0 depend on these estimated values $\left({ }_{0}^{i} \mathbf{h}\right)$, the optimum of Level-0 can shift for different estimated values $\left({ }_{0}^{i} \mathbf{h}\right.$ ). Determining an (optimal) value for the coupling variable vector ${ }_{0}^{i} \mathbf{h}$ is accomplished via adding the coupling variable vector to the optimization problem in the form of design variables. The physical responses ${ }_{i}^{0} \mathcal{H}\left({ }^{0} \mathbf{r}\right)={ }_{i}^{0} \mathbf{h}$ that are mapped from Level- 0 to Level- 1 are not added as design variables. These variables $\left({ }_{i}^{0} \mathbf{h}\right)$ are accounted for in the analysis of the Level-1 element because they are directly mapped (top-down hierarchic decomposition). 
Equation 4 is now modified and is mathematically expressed as:

$$
\begin{aligned}
& \min _{\mathbf{x},{ }_{0}^{i} \mathbf{h}} \quad{ }^{0} v_{f}\left({ }_{0}^{i} \mathbf{h},{ }_{i}^{0} \mathbf{z},{ }^{0} \mathbf{x},{ }^{0} \mathbf{r}\left({ }_{i}^{0} \mathbf{z},{ }^{0} \mathbf{x},{ }_{0}^{i} \mathbf{h}\right)\right)+\sum_{i=1}^{3}{ }^{i} v_{f}\left({ }_{0}^{i} \mathbf{z},{ }^{i} \mathbf{x},{ }^{i} \mathbf{r}\left({ }_{0}^{i} \mathbf{z},{ }^{i} \mathbf{x},{ }_{i}^{0} \mathbf{h}\right)\right) \\
& \text { s.t. } \quad{ }^{0} \mathbf{v}_{g}\left({ }_{0}^{i} \mathbf{h},{ }_{i}^{0} \mathbf{z},{ }^{0} \mathbf{x},{ }^{0} \mathbf{r}\left({ }_{i}^{0} \mathbf{z},{ }^{0} \mathbf{x},{ }_{0}^{i} \mathbf{h}\right)\right) \leq \mathbf{0} \\
& { }^{i} \mathbf{v}_{g}\left({ }_{0}^{i} \mathbf{z},{ }^{i} \mathbf{x},{ }^{i} \mathbf{r}\left({ }_{0}^{i} \mathbf{z},{ }^{i} \mathbf{x},{ }_{i}^{0} \mathbf{h}\right)\right) \leq \mathbf{0} \quad i=1, \ldots, 3 \\
& { }^{0} \mathbf{v}_{h}\left({ }_{0}^{i} \mathbf{h},{ }_{i}^{0} \mathbf{z},{ }^{0} \mathbf{x},{ }^{0} \mathbf{r}\left({ }_{i}^{0} \mathbf{z},{ }^{0} \mathbf{x},{ }_{0}^{i} \mathbf{h}\right)\right)=\mathbf{0} \\
& { }^{i} \mathbf{v}_{h}\left({ }_{0}^{i} \mathbf{z},{ }^{i} \mathbf{x},{ }^{i} \mathbf{r}\left({ }_{0}^{i} \mathbf{z},{ }^{i} \mathbf{x},{ }_{i}^{0} \mathbf{h}\right)\right)=\mathbf{0} \\
& \text { where } \\
& { }^{0} \mathbf{x},{ }^{0} \mathbf{r} \quad \text { top-element level variables } \\
& { }^{i} \mathbf{x},{ }^{i} \mathbf{r} \quad \text { element level variables } \\
& . \mathbf{h} \quad \text { coupling variables } \\
& . \mathbf{z} \quad \text { shared design variables } \\
& \mathbf{x}=\left[{ }^{0} \mathbf{x},{ }_{i}^{0} \mathbf{z},{ }^{i} \mathbf{x},{ }_{0}^{i} \mathbf{z}\right] \\
& { }_{i}^{0} \mathcal{H}\left({ }^{0} \mathbf{r}\right)={ }_{i}^{0} \mathbf{h} \\
& { }_{0}^{i} \mathbf{c}={ }_{0}^{i} \mathcal{H}\left({ }^{i} \mathbf{r}\right)-{ }_{0}^{i} \mathbf{h}=0 \\
& { }_{i}^{0} \mathbf{c}_{z}={ }_{i}^{0} \mathbf{z}-{ }_{0}^{i} \mathbf{z}=0 \\
& { }_{0}^{i} \underline{\mathbf{h}} \leq{ }_{0}^{i} \mathbf{h} \leq{ }_{0}^{i} \overline{\mathbf{h}}
\end{aligned}
$$

Notice that additional design variables $\left({ }_{0}^{i} \mathbf{h}\right)$ are introduced and consistency constraints $\left({ }_{0}^{i} \mathbf{c}\right)$ are present that take into account the coupling between Level-1 and Level-0. Furthermore, consistency constraints are present that relate ${ }_{i}^{0} \mathbf{z}$ to ${ }_{0}^{i} \mathbf{z}$. Finally, lower $\left({ }_{0}^{i} \underline{\mathbf{h}}\right)$ and upper $\left({ }_{0}^{i} \overline{\mathbf{h}}\right)$ bounds on the coupling variables ${ }_{0}^{i} \mathbf{h}$ are added that correspond to physically significant output of Level- 1 that is now constrained via the consistency constraints.

The optimization problem presented in Equation 4 is an example of optimization problems with embedded hierarchy. In general, four typical patterns can be distinguished in the problem matrix of optimization problems with embedded hierarchy. These four characteristic coupling patterns are shown in Figure 13 For brevity of Figure 13 shared design variables $\mathbf{z}$ are not explicitly shown, however these enter the problem matrix similar to the coupling variables. A dark colored block indicates that the functions (horizontally) depend on the design variables and responses (vertically). A light colored block indicates a weak dependence and a white colored block indicates dependence is absent.

In Figure 13, pattern (a) illustrates a problem matrix where the objective function only depends on the top-level element. Pattern (b) illustrates the case where a small number of coupling variables couples the objective and constraint functions of all the levels. Pattern (c) illustrates a problem where the design variables and/or physical responses are shared over multiple levels. Pattern (d) illustrates a problem where the objective function and the constraints depend on all the design variables and physical responses of all the elements.

Multi-level or multi-disciplinary optimization methods are defined for optimization problems that belong to one of these four problem matrices. These multi-level or multi-disciplinary optimization methods coordinate data from the individual elements such that the optimal solution to the entire hierarchy is found. Research into finding optimal problem decompositions to reduce coordination has been conducted by, e.g. Bloebaum (1995), ${ }^{19}$ Altus et al.(1996), ${ }^{11}$ Chen and Li (2005) ${ }^{23}$ and Allison et al.(2009). ${ }^{9}$ Coordination of individual elements is discussed in the next section.

\section{Coordination}

Solutions of decomposed elements require coordination, such that the individual solutions combined lead to the solution for the entire hierarchy. Coordination of interaction between elements involves decision making. Consider two individual element optimizations transferring data, see Figure 14(a). The origin of this data can be, e.g., physical responses (r) such as shown in Figure 14(a) or shared design variables (z). This data cannot be arbitrarily send from one element to the neighboring element. Therefore, decisions on which data to send and in what order are necessary.

There are three types of decisions that can be distinguished: ${ }^{63}$

1. separable decisions;

2. inseparable decisions; 

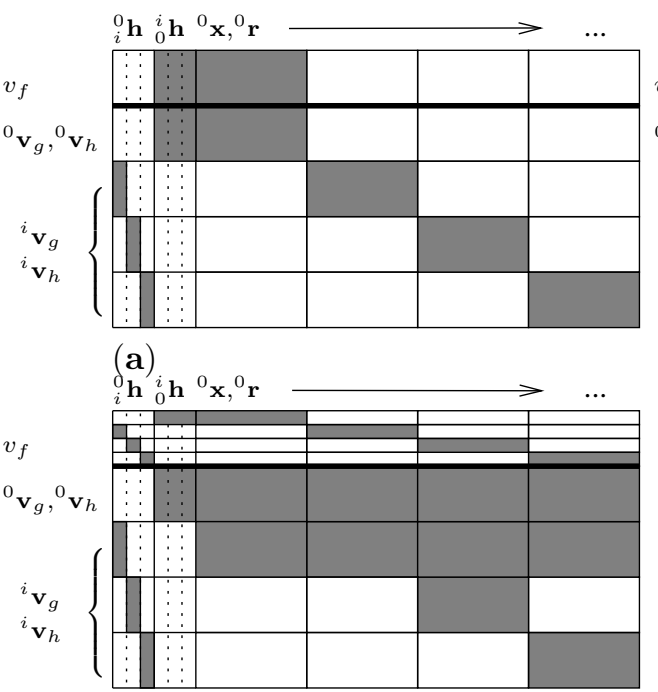

(c)

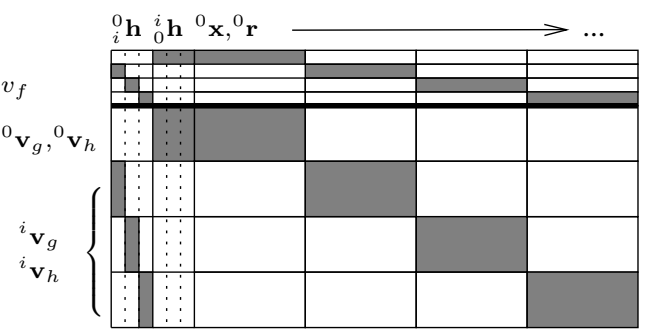

(b)

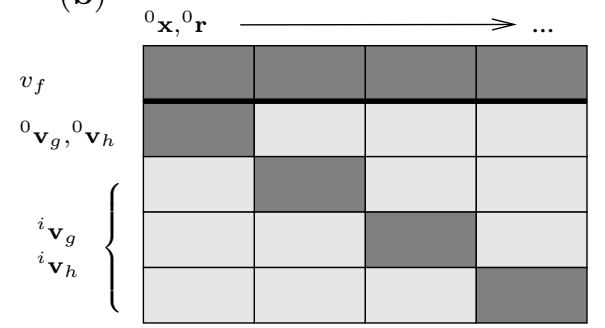

(d)

Figure 13. Four typical patterns can be distinguished in the problem matrix. A dark colored block indicates that the functions (horizontally) depend on the design variables and responses (vertically). A light colored block indicates a weak dependence and a white colored block indicates dependence is absent. (a) A hierarchy where the objective function depends on the top-level design variables ${ }^{0} \mathrm{x}$ and physical responses ${ }^{0} \mathrm{r}$ and on the coupling variables ${ }_{0}^{i} \mathrm{~h}$. The lower level elements do not have an individual objective function. (b) A hierarchy where the objective function and constraint functions share a small number of coupling variables. Level-0 as well as elements present at Level-1 contribute to the minimization of the additively separable objective function $v_{f}$. (c) A hierarchy where the design variables and physical responses of different elements contribute to the constraint functions of neighboring elements. Some of the elements in lower levels depend on physical responses and/or design variables from neighboring elements. (d) No clear hierarchy can be distinguished. The objective function as well as the constraint functions depend on the physical responses and design variables from all the elements. Either Global Sensitivity Equations (GSE) ${ }^{6}$ are required to separate the elements or relaxation of the consistency constraints between the elements is necessary.

3. inseparable and coupled decisions.

The first type of decisions, the separable decisions are shown in Figure 14(b). These type of decisions occur in a multi-level hierarchy where the update of information of one element does not depend on the data that is send to neighboring elements. This is typically the case for elements on the same level that are not coupled to each other. Hence, after solving the parent element (Level-0), the updates of child elements (Level-1) are independent from one another.

The second type of decisions are inseparable decisions shown in Figure 14(c). The output of one element is send to the input of the neighboring element. These decisions are done sequentially and are the result of top-down or bottom-up decomposition schemes. No operations are performed inside the coordinator block and no changes are made to the Level-0 optimization problem after it has finished. This type of coordination fails in cases where the Level-0 element prescribes physical responses (i.e. ${ }_{0}^{1} \mathbf{h}$ ) or shared design variable vectors (i.e. ${ }_{1}^{0} \mathbf{z}={ }_{0}^{1} \mathbf{z}$ ) that the Level-1 element is unable to meet. Either due to restrictions on design variables (i.e. lower bounds $\underline{\mathbf{x}}, \underline{\mathbf{z}}$ and/or upper bounds $\overline{\mathbf{x}}, \overline{\mathbf{z}}$ ) and/or physical restrictions on the Level-1 element. Therefore, the Level-0 optimization problem requires additional information via, e.g., Optimum Sensitivity Analysis ${ }^{49,54,73}$ or a copy of the relaxed consistency function. ${ }^{41}$

The third type of decisions are the inseparable and coupled decisions shown in Figure 14(d). The output of one element is the input of the neighboring element and vice versa. These decisions are done sequentially via iterating or done concurrently through optimization.

To illustrate how inseparable decisions are tackled via coordination of a multi-level hierarchic optimization 


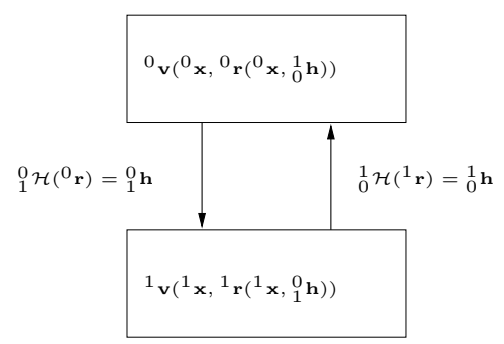

(a)

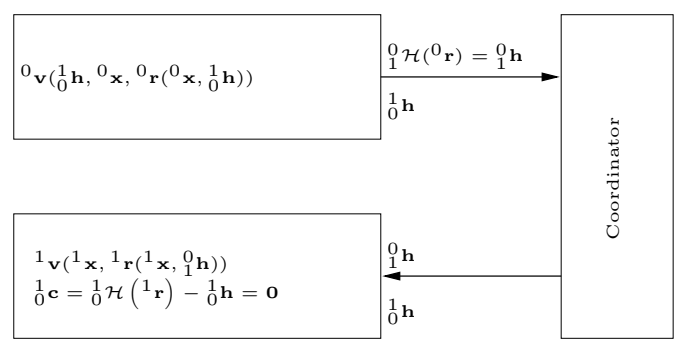

(c)

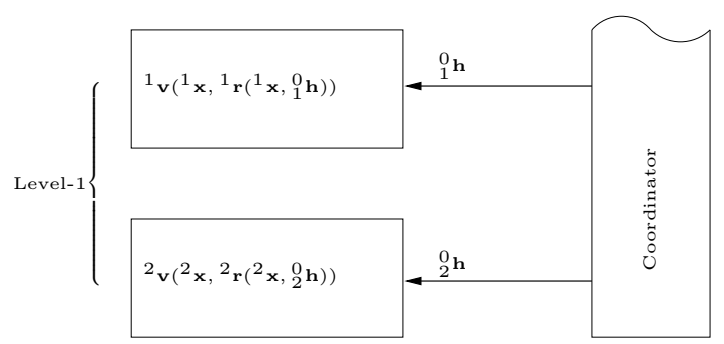

(b)

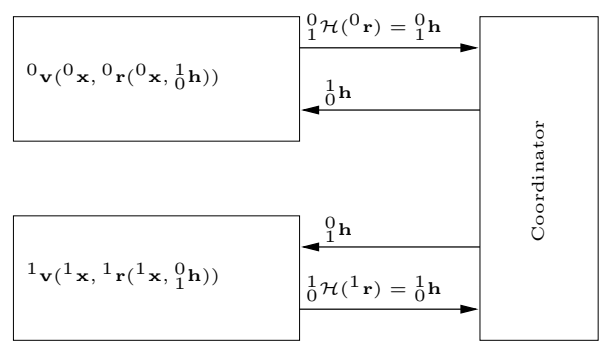

(d)

Figure 14. (a) Information exchange between two individual coupled optimization problems. Coordination of the data that is send between the two elements is necessary. (b) Separable decisions, the optimization problems are not coupled. Hence, the data for the first element at Level-1 does not change the outcome of the neighboring second element at Level-1. (c) Inseparable decisions, the optimization problems are coupled and data is send from one element to the neighboring element. (d) Inseparable coupled decisions require iteration. The solution of both elements depends on the solution of the neighboring element.

problem the following all-in-one optimization problem is considered:

$$
\begin{aligned}
& \min _{\cdots \mathbf{x},: . \mathbf{z}} v_{f}={ }^{0} v_{f}\left({ }^{0} \mathbf{x},{ }_{i}^{0} \mathbf{z},{ }^{0} \mathbf{r}\left({ }^{0} \mathbf{x},{ }_{i}^{0} \mathbf{z},{ }_{0}^{i} \mathbf{h}\right)\right)+{ }^{i} v_{f}\left({ }^{i} \mathbf{x},{ }_{0}^{i} \mathbf{z},{ }^{i} \mathbf{r}\left({ }^{i} \mathbf{x},{ }_{0}^{i} \mathbf{z},{ }_{i}^{0} \mathbf{h}\right)\right) \\
& v_{g}= \begin{cases}{ }^{0} v_{g}\left({ }^{0} \mathbf{x},{ }_{i}^{0} \mathbf{z},{ }^{0} \mathbf{r}\left({ }^{0} \mathbf{x},{ }_{i}^{0} \mathbf{z},{ }_{0}^{i} \mathbf{h}\right)\right) \leq 0 \\
{ }^{i} v_{g}\left({ }^{i} \mathbf{x},{ }_{0}^{i} \mathbf{z},{ }^{i} \mathbf{r}\left({ }^{i} \mathbf{x},{ }_{0}^{i} \mathbf{z},{ }_{i}^{0} \mathbf{h}\right)\right) \leq 0\end{cases} \\
& v_{h}= \begin{cases}{ }^{0} v_{h}\left({ }^{0} \mathbf{x},{ }_{i}^{0} \mathbf{z},{ }^{0} \mathbf{r}\left({ }^{0} \mathbf{x},{ }_{i}^{0} \mathbf{z},{ }_{0}^{i} \mathbf{h}\right)\right)=0 \\
{ }^{i} v_{h}\left({ }^{i} \mathbf{x},{ }_{0}^{i} \mathbf{z},{ }^{i} \mathbf{r}\left({ }^{i} \mathbf{x},{ }_{0}^{i} \mathbf{z},{ }_{i}^{0} \mathbf{h}\right)\right)=0\end{cases} \\
& \cdot \underline{\mathbf{x}} \leq \cdots \mathbf{x} \leq \cdot \overline{\mathbf{x}} \quad ; \quad . \quad \underline{\mathbf{z}} \leq \cdots \mathbf{z} \leq \cdot . \overline{\mathbf{z}} ; \\
& \text { where } \quad{ }_{i}^{0} \mathcal{H}\left({ }^{0} \mathbf{r}\right)={ }_{i}^{0} \mathbf{h} ; \quad{ }_{0}^{i} \mathcal{H}\left({ }^{i} \mathbf{r}\right)={ }_{0}^{i} \mathbf{h} ; \quad{ }_{i}^{0} \mathbf{z}={ }_{0}^{i} \mathbf{z} .
\end{aligned}
$$

Hence, coupling is present between the physical responses $\left({ }^{0} \mathbf{r},{ }^{i} \mathbf{r}\right)$ and coupling is present due to shared design variables $\left({ }_{i}^{0} \mathbf{z},{ }_{0}^{i} \mathbf{z}\right)$. The notation is applied according to the hierarchy that is identified within the optimization problem.

Equation [6 is distributed over two levels. A single optimization problem is located at the top-level (Level-0) which is coupled to the individual optimization problems located one level lower (Level-1). This is accomplished via a top-down hierarchic decomposition (Figure 12), hence additional consistency constraints are introduced to Equation 6. The hierarchical top-down decomposed problem is split into a Level-0 optimization problem that is mathematically expressed as:

$$
\begin{array}{ll}
\min _{{ }^{0} \mathbf{x},{ }_{i}^{0} \mathbf{z},{ }_{0}^{i} \mathbf{h}} & { }^{0} v_{f}\left({ }_{0}^{i} \mathbf{h},{ }^{0} \mathbf{x},{ }_{i}^{0} \mathbf{z},{ }^{0} \mathbf{r}\left({ }^{0} \mathbf{x},{ }_{i}^{0} \mathbf{z},{ }_{0}^{i} \mathbf{h}\right)\right) \\
\text { s.t. } & { }^{0} \mathbf{v}_{g}\left({ }^{0} \mathbf{x},{ }_{i}^{0} \mathbf{z},{ }^{0} \mathbf{r}\left({ }_{0}^{i} \mathbf{h},{ }^{0} \mathbf{x},{ }_{i}^{0} \mathbf{z},{ }_{0}^{i} \mathbf{h}\right)\right) \leq 0 \\
& { }^{0} \mathbf{v} h\left({ }^{0} \mathbf{x},{ }_{i}^{0} \mathbf{z},{ }^{0} \mathbf{r}\left({ }_{0}^{i} \mathbf{h},{ }_{0}^{0} \mathbf{x},{ }_{i}^{0} \mathbf{z},{ }_{0}^{i} \mathbf{h}\right)\right)=0 \\
& { }^{0} \underline{\mathbf{x}} \leq{ }^{0} \mathbf{x} \leq{ }^{0} \overline{\mathbf{x}} ; \quad{ }^{0} \mathbf{z} \leq{ }_{i}^{0} \mathbf{z} \leq{ }_{i}^{0} \overline{\mathbf{z}} \quad ; \quad{ }_{0}^{i} \underline{\mathbf{h}} \leq{ }_{0}^{i} \mathbf{h} \leq{ }_{0}^{i} \overline{\mathbf{h}} ; \\
\text { where } & { }_{i}^{0} \mathcal{H}\left({ }^{0} \mathbf{r}\right)={ }_{i}^{0} \mathbf{h} .
\end{array}
$$

The physical responses $\left({ }^{0} \mathbf{r}\right)$ are mapped directly onto the Level- 1 optimization problems as well as the shared design variables $\left({ }_{i}^{0} \mathbf{z}\right)$. Furthermore, coupling variables ${ }_{0}^{i} \mathbf{h}$ are chosen as design variables for the Level0 optimization problem. Hence, the two levels are decoupled via the consistency constraints on the physical 
coupling that are assigned to Level-1 and via consistency constraints on the shared design variables that are assigned to Level-1.

The Level-1 optimization problems are mathematically expressed as:

$$
\begin{array}{ll}
\min _{\mathbf{i},{ }_{0}^{i} \mathbf{z}} & { }^{i} v_{f}\left({ }^{i} \mathbf{x},{ }^{i} \mathbf{r}\left({ }^{i} \mathbf{x},{ }_{0}^{i} \mathbf{z},{ }_{0}^{i} \mathbf{h}\right)\right) \\
\text { s.t. } & { }^{i} \mathbf{v}_{g}\left({ }^{i} \mathbf{x},{ }_{0}^{i} \mathbf{z},{ }^{0} \mathbf{r}\left({ }^{0} \mathbf{x},{ }_{0}^{i} \mathbf{z},{ }_{0}^{i} \mathbf{h}\right)\right) \leq 0 \\
& { }^{i} \mathbf{v}_{h}\left({ }^{i} \mathbf{x},{ }_{0}^{i} \mathbf{z},{ }^{0} \mathbf{r}\left({ }^{0} \mathbf{x},{ }_{0}^{i} \mathbf{z},{ }_{0}^{i} \mathbf{h}\right)\right)=0 \\
& { }^{i} \mathbf{v}_{{ }^{i}} c\left({ }_{0}^{i} \mathbf{c}\left({ }^{i} \mathbf{r}\right)\right)={ }_{0}^{i} \mathcal{H}\left({ }^{i} \mathbf{r}\right)-{ }_{0}^{i} \mathbf{h}=0 \\
& { }^{i} \mathbf{v}_{i}^{0} c_{z}\left({ }_{i}^{0} \mathbf{c}_{z}\left({ }_{0}^{i} \mathbf{z}\right)\right)={ }_{i}^{0} \mathbf{z}-{ }_{0}^{i} \mathbf{z}=0 \\
& { }^{i} \underline{\mathbf{x}} \leq{ }^{i} \mathbf{x} \leq{ }^{i} \overline{\mathbf{x}} ; \quad ; \quad{ }_{0}^{i} \underline{\mathbf{z}} \leq{ }_{0}^{i} \mathbf{z} \leq{ }_{0}^{i} \overline{\mathbf{z}} .
\end{array}
$$

Consistency is maintained via the consistency equations $\left(\mathbf{c}, \mathbf{c}_{z}\right)$.

The above problem formulation (Equations 7 and 8 ) has the problem that, if values of ${ }_{i}^{0} \mathcal{H}\left({ }^{0} \mathbf{r}\right),{ }_{0}^{i} \mathbf{h}$ and ${ }_{i}^{0} \mathbf{z}$ are determined within the Level-0 problem that do not correspond to feasible solutions within the Level-1 optimization problems, there is no answer to the multi-level optimization problem. Various researchers (e.g. Vanderplaats et al. ${ }^{84}$ and $\mathrm{Kirsch}^{42}$ ) reported this problem. A solution to this problem depends on the decomposition formulation chosen and is discussed in the next two sections.

\section{IV.A. Equality-based coordination}

To overcome the problem that leads to infeasible elements within the hierarchy, two separate approaches exist depending on the decomposition formulation chosen. In case strong (equality) consistency constraints are formulated the solution is to add additional information on the Level-1 optimization problems to the Level-0 optimization problem.

The Level-0 optimization problem in Equation 7 is modified and is mathematically expressed as:

$$
\begin{array}{ll}
\min _{{ }^{0} \mathbf{x},,_{i}, \mathbf{z},{ }_{0}^{i}} \mathbf{h} & { }^{0} v_{f}\left({ }_{0}^{i} \mathbf{h},{ }^{0} \mathbf{x},{ }_{i}^{0} \mathbf{z},{ }^{0} \mathbf{r}\left({ }^{0} \mathbf{x},{ }_{i}^{0} \mathbf{z},{ }_{0}^{i} \mathbf{h}\right)\right) \\
\text { s.t. } & { }^{0} \mathbf{v}_{g}\left({ }_{0}^{i} \mathbf{h},{ }^{0} \mathbf{x},{ }_{i}^{0} \mathbf{z},{ }^{0} \mathbf{r}\left({ }^{0} \mathbf{x},{ }_{i}^{0} \mathbf{z},{ }_{0}^{i} \mathbf{h}\right)\right) \leq 0 \\
& { }^{0} \mathbf{v}_{h}\left({ }_{0}^{i} \mathbf{h},{ }^{0} \mathbf{x},{ }_{i}^{0} \mathbf{z},{ }^{0} \mathbf{r}\left({ }^{0} \mathbf{x},{ }_{i}^{0} \mathbf{z},{ }_{0}^{i} \mathbf{h}\right)\right)=0 \\
& { }^{0} \mathbf{v}_{a}=f\left({ }_{0}^{i} \mathbf{z},{ }_{0}^{i} \mathbf{h},{ }^{0} \mathbf{r}\left({ }^{0} \mathbf{x},{ }_{i}^{0} \mathbf{z},{ }_{0}^{i} \mathbf{h}\right)\right) \leq 0 \\
& { }^{0} \underline{\mathbf{x}} \leq \mathbf{x} \leq{ }^{0} \overline{\mathbf{x}} ; \quad ; \quad{ }_{0}^{i} \mathbf{z} \leq{ }_{0}^{i} \mathbf{z} \leq{ }_{0}^{i} \overline{\mathbf{z}} ; \quad ; \quad \underline{\mathbf{h}} \leq{ }_{0}^{i} \mathbf{h} \leq{ }_{0}^{i} \overline{\mathbf{h}}, \\
\text { where } & { }_{i}^{0} \mathcal{H}\left({ }^{0} \mathbf{r}\right)={ }_{i}^{0} \mathbf{h} .
\end{array}
$$

Hence, additional functions $\left(\mathbf{v}_{a}\right)$ are present that take into account the behavior of neighboring elements. No changes are made to the Level-1 problem formulation. Therefore, a mathematical expression of Level-1 problems is equal to the expression presented in Equation 8 .

In summary, the problem description of an element that is part of a hierarchy decomposed via equality consistency equations is written as:

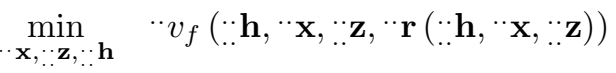

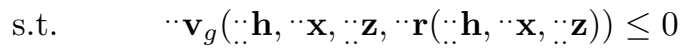

$$
\begin{aligned}
& \cdot \mathbf{v}_{h}(\cdot . \mathbf{h}, \cdot \cdot \mathbf{x}, \cdot . \mathbf{z}, \cdot \cdot \mathbf{r}(\cdot . \cdot \mathbf{h}, \cdot \cdot \mathbf{x}, \cdot . \cdot \mathbf{z}))=0 \\
& \cdot \mathbf{v} . . c(. . \cdot \mathbf{c}(\cdot \mathbf{r}, . . \cdot \mathbf{h}))=0 \\
& \cdot \mathbf{v}_{\because . . c_{z}(. .}\left(\mathbf{c}_{z}(. . \mathrm{z}, . . \mathrm{z})\right)=0 \\
& { }^{*} \mathbf{v}_{a}(. . . \mathbf{h}, . . \mathbf{z}, \cdot \mathbf{r}) \leq 0 \\
& \because \underline{\mathbf{x}} \leq \cdots \mathbf{x} \leq \cdot \overline{\mathbf{x}} \quad ; \quad . \quad \underline{\mathbf{z}} \leq . . \mathrm{z} \leq . . \overline{\mathbf{z}} \quad ; \quad . . \underline{\mathbf{h}} \leq . . \mathrm{h} \leq . . \overline{\mathbf{h}} .
\end{aligned}
$$

The consistency constraints $\left(\mathbf{v}_{c}\right)$ take into account the consistency with: higher elements in the hierarchy (top-down decomposition); lower elements in the hierarchy (bottom-up decomposition); and/or neighboring elements (non-hierarchic decomposition). Likewise, the constraints that approximate neighboring elements $\left(v_{a}\right)$ take into account the behavior of elements: located lower in the hierarchy (top-down decomposition); located higher in the hierarchy (bottom-up decomposition); and/or neighboring elements (non-hierarchic decomposition). 
Approximations are constructed via a two step process. First, the type of information that is required from neighboring elements is chosen. Second, a means of constructing the function that approximates the data from the neighboring element is formulated.

Data from neighboring elements that is approximated in the current element consists of:

- the value of objective function and/or constraint functions that are assigned to neighboring elements. This data informs the current element whether an increas/decrease of the objective function in neighboring elements or constraint violations in neighboring elements are to be expected due to changes in the current element.

- a measure of constraint margin that informs the current element how much "design space" is left in the neighboring elements before the neighboring elements become infeasible due to changes in the current element.

- the physical coupling that is mapped from a neighboring element onto the current element.

- the value of objective function and/or constraint functions that are shared with neighboring elements. This data accounts for constraints that are shared and are satisfied in neighboring elements. Therefore, in the current element they can be neglected and vice versa.

The behavior of a neighboring element is modeled via a function $\left(\mathbf{v}_{a}\right)$ that depends on coupling data from the current element. To construct an approximation to the neighboring element(s) various approaches exist:

- linearization requires sensitivities, e.g., via optimum sensitivity derivatives ${ }^{14}$ global sensitivity equations, ${ }^{66}$ amongst others.

- response surface approximations. ${ }^{36,72,86}$

Linearization may consist of linearizing constraints ${ }^{16}$ in Level-1 with respect to the interaction between elements, hence ${ }^{\prime} \cdot \boldsymbol{H}(\cdot \mathbf{r}), \cdot \cdot \mathbf{h}$ and ${ }^{\prime} \mathbf{z}$. In addition, these linearized constraints are added to the Level0 optimization problem. ${ }^{84}$ In case non-hierarchic decomposition is applied the so-called Global Sensitivity Equations ${ }^{62,66,67}$ are evaluated to obtain the necessary sensitivity information for the shared constraints. ${ }^{64,70}$

Linearization of coupled optimization functions may require a small step size to update the coupling data between elements. ${ }^{61}$ Increasing accuracy of the linearized coupling is accomplished via adding higher order information to the linearization. ${ }^{55,56}$ Another approach that is used to approximate behavior of neighboring elements is via response surfaces for individual elements. ${ }^{60,71,72}$

Constraint margin approaches create design freedom via the Level-1 optimization problems such that the Level-0 optimization problem can search within a design space that is feasible. Sobieszczanski-Sobieski et al. $(1985,1987)^{75,76}$ applied a technique that captures multiple constraints into a single function. ${ }^{12,68,69}$ This single function is added to the objective function of a Level-1 element. The objective of the Level1 optimization problem is to push the Level-1 design points as far as possible into the feasible domain. Haftka and Watson $(2005)^{36,37,50,59}$ developed a similar strategy where slack variables were introduced to the constraints of neighboring elements that directed the constraints as far away as possible from values where the constraints become critical. In addition, Haftka and Watson provide a mathematical justification of the method.

\section{IV.B. Relaxation-based coordination}

Relaxation based approaches overcome the problem of infeasible elements via a copy of the relaxed consistency constraints. To demonstrate the procedure Equations 7 and 8 are repeated. However, the consistency constraints are now relaxed via, e.g., Lagrangian relaxation. No changes to the Level-0 optimization problem are made.

The Level-0 optimization problem is mathematically expressed as:

$$
\begin{array}{ll}
\min _{{ }^{0} \mathbf{x},{ }_{i}^{0} \mathbf{z},{ }_{0}^{i} \mathbf{h}} & { }^{0} v_{f}\left({ }_{0}^{i} \mathbf{h},{ }^{0} \mathbf{x},{ }_{i}^{0} \mathbf{z},{ }^{0} \mathbf{r}\left({ }^{0} \mathbf{x},{ }_{i}^{0} \mathbf{z},{ }_{0}^{i} \mathbf{h}\right)\right) \\
\text { s.t. } & { }^{0} \mathbf{v}_{g}\left({ }_{0}^{i} \mathbf{h},{ }^{0} \mathbf{x},{ }_{i}^{0} \mathbf{z},{ }^{0} \mathbf{r}\left({ }^{0} \mathbf{x},{ }_{i}^{0} \mathbf{z},{ }_{0}^{i} \mathbf{h}\right)\right) \leq 0 \\
& { }^{0} \mathbf{v} h\left({ }_{0}^{i} \mathbf{h},{ }^{0} \mathbf{x},{ }_{i}^{0} \mathbf{z},{ }^{0} \mathbf{r}\left({ }^{0} \mathbf{x},{ }_{i}^{0} \mathbf{z},{ }_{0}^{i} \mathbf{h}\right)\right)=0 \\
& { }^{0} \underline{\mathbf{x}} \leq{ }^{0} \mathbf{x} \leq{ }^{0} \overline{\mathbf{x}} ; \quad{ }^{0} \mathbf{z} \leq{ }_{i}^{0} \mathbf{z} \leq{ }_{i}^{0} \overline{\mathbf{z}} \quad ; \quad{ }_{0}^{i} \underline{\mathbf{h}} \leq{ }_{0}^{i} \mathbf{h} \leq{ }_{0}^{i} \overline{\mathbf{h}} \\
\text { where } & { }_{i}^{0} \mathcal{H}\left({ }^{0} \mathbf{r}\right)={ }_{i}^{0} \mathbf{h} .
\end{array}
$$


The Level-1 optimization problem is modified and is mathematically expressed as:

$$
\begin{aligned}
& \min _{{ }^{i} \mathbf{x},{ }_{0}^{i} \mathbf{z}}{ }^{i} v_{f}\left({ }^{i} \mathbf{x},{ }^{i} \mathbf{r}\left({ }^{i} \mathbf{x},{ }_{0}^{i} \mathbf{z},{ }_{0}^{i} \mathbf{h}\right)\right)+\sum{ }^{i} v_{0} c\left({ }_{0}^{i} \mathbf{c}\left({ }^{i} \mathbf{r}\right)\right)+\sum{ }^{i} v_{i} c_{z}\left({ }_{i}^{0} \mathbf{c}_{z}\left({ }_{0}^{i} \mathbf{z}\right)\right) \\
& \text { s.t. } \quad{ }^{i} \mathbf{v}_{g}\left({ }^{i} \mathbf{x},{ }_{0}^{i} \mathbf{z},{ }^{i} \mathbf{r}\left({ }^{i} \mathbf{x},{ }_{0}^{i} \mathbf{z},{ }_{0}^{i} \mathbf{h}\right)\right) \leq 0 \\
& { }^{i} \mathbf{v}_{h}\left({ }^{i} \mathbf{x},{ }_{0}^{i} \mathbf{z},{ }^{i} \mathbf{r}\left({ }^{i} \mathbf{x},{ }_{0}^{i} \mathbf{z},{ }_{0}^{i} \mathbf{h}\right)\right)=0 \\
& { }^{i} \underline{\mathbf{x}} \leq{ }^{i} \mathbf{x} \leq{ }^{i} \overline{\mathbf{x}} \quad ; \quad{ }_{0}^{i} \underline{\underline{z}} \leq{ }_{0}^{i} \mathbf{z} \leq{ }_{0}^{i} \overline{\mathbf{z}} ; \\
& \text { where }{ }^{i} v_{0}^{i} c\left({ }_{0}^{i} \mathbf{c}\left({ }^{i} \mathbf{r}\right)\right)={ }_{0}^{i} \boldsymbol{\lambda}^{T}\left({ }_{0}^{i} \mathcal{H}\left({ }^{i} \mathbf{r}\right)-{ }_{0}^{i} \mathbf{h}\right) \text {, } \\
& { }^{i} v_{i} c_{z}\left({ }_{i}^{0} \mathbf{c}_{z}\left({ }_{0}^{i} \mathbf{z}\right)\right)={ }_{0}^{i} \boldsymbol{\lambda}^{T}\left({ }_{i}^{0} \mathbf{z}-{ }_{0}^{i} \mathbf{z}\right) \text {. }
\end{aligned}
$$

In Equation 12 the consistency constraints $\left(\mathbf{c}\right.$ and $\mathbf{c}_{z}$ ) are relaxed.

The problem formulation in Equations 111 and 12 suffers from the same problem as mentioned in the introduction of Section IV. Hence, infeasible Level-1 elements can be present due to coupling data sent from the Level-0 element to the Level-1 elements. Therefore, additional information about the Level-1 optimization is added to the Level-0 optimization via a copy of the relaxed consistency constraints.

The modified Level-0 optimization problem is mathematically expressed as:

$$
\begin{aligned}
& \min _{{ }^{0} \mathbf{x},{ }_{i} \mathbf{z},{ }_{0}^{i} \mathbf{h}}{ }^{0} v_{f}\left({ }_{0}^{i} \mathbf{h},{ }^{0} \mathbf{x},{ }_{i}^{0} \mathbf{z},{ }^{0} \mathbf{r}\left({ }^{0} \mathbf{x},{ }_{i}^{0} \mathbf{z},{ }_{0}^{i} \mathbf{h}\right)\right)+\sum \sum{ }^{0} v_{0}^{i} c\left({ }_{0}^{i} \mathbf{c}\left({ }_{0}^{i} \mathbf{h}\right)\right)+\sum{ }^{0} v_{i}^{0} c_{z}\left({ }_{i}^{0} \mathbf{c}_{z}\left({ }_{i}^{0} \mathbf{z}\right)\right) \\
& \text { s.t. } \quad{ }^{0} \mathbf{v}_{g}\left({ }_{0}^{i} \mathbf{h},{ }^{0} \mathbf{x},{ }_{i}^{0} \mathbf{z},{ }^{0} \mathbf{r}\left({ }^{0} \mathbf{x},{ }_{i}^{0} \mathbf{z},{ }_{0}^{i} \mathbf{h}\right)\right) \leq 0 \\
& { }^{0} \mathbf{v}_{h}\left({ }_{0}^{i} \mathbf{h},{ }^{0} \mathbf{x},{ }_{i}^{0} \mathbf{z},{ }^{0} \mathbf{r}\left({ }^{0} \mathbf{x},{ }_{i}^{0} \mathbf{z},{ }_{0}^{i} \mathbf{h}\right)\right)=0 \\
& { }_{i}^{0} \mathcal{H}\left({ }^{0} \mathbf{r}\right)={ }_{i}^{0} \mathbf{h} ; \quad{ }_{i}^{0} \mathbf{z}={ }_{0}^{i} \mathbf{z} \\
& { }^{0} \underline{\mathbf{x}} \leq{ }^{0} \mathbf{x} \leq{ }^{0} \overline{\mathbf{x}} \quad ; \quad{ }_{i}^{0} \underline{\mathbf{z}} \leq{ }_{i}^{0} \mathbf{z} \leq{ }_{i}^{0} \overline{\mathbf{z}} \quad ; \quad{ }_{0}^{i} \underline{\mathbf{h}} \leq{ }_{0}^{i} \mathbf{h} \leq{ }_{0}^{i} \overline{\mathbf{h}} ; \\
& \text { where } \quad{ }^{0} \mathbf{v}_{0}{ }_{0} c\left({ }_{0}^{i} \mathbf{c}\left({ }_{0}^{i} \mathbf{h}\right)\right)={ }_{0}^{i} \boldsymbol{\lambda}^{T}\left({ }_{0}^{i} \mathcal{H}\left({ }^{i} \mathbf{r}\right)-{ }_{0}^{i} \mathbf{h}\right) \text {; } \\
& { }^{0} \mathbf{V}_{i}^{0} c_{z}\left({ }_{i}^{0} \mathbf{c}_{z}\left({ }_{i}^{0} \mathbf{z}\right)\right)={ }_{0}^{i} \boldsymbol{\lambda}^{T}\left({ }_{i}^{0} \mathbf{z}-{ }_{0}^{i} \mathbf{z}\right) \text {. }
\end{aligned}
$$

The copied relaxed consistency constraints are added to the objective function value. The Level-1 optimization problem remains the same, i.e., as in Equation 12 The Lagrange multipliers are kept constant during the individual element optimizations. These Lagrange multipliers are only updated via decisions made via the coordinator. Furthermore, in Equations 13 and 12 the Lagrange multipliers are equal. Depending on the coordinator these parameters can be different.

In summary, an element optimization problem that is coupled to neighboring elements and for which this coupling is relaxed is mathematically expressed as:

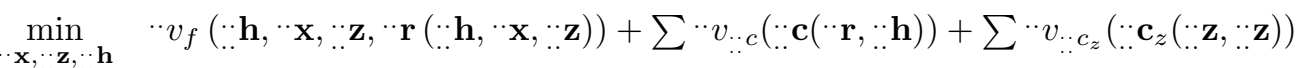

$$
\begin{aligned}
& \text { s.t. } \quad \cdot v_{g}(. . \mathbf{h}, \cdot \mathbf{x}, . . \mathbf{z}, \cdot \mathbf{r}(\cdot . \mathbf{h}, \cdot \mathbf{x}, \cdot . \mathbf{z})) \leq 0
\end{aligned}
$$

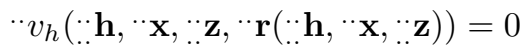

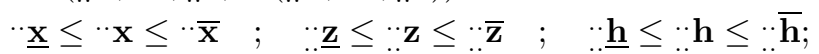

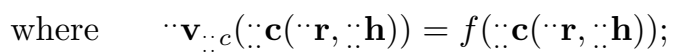

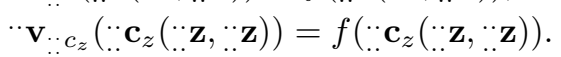

The relaxed consistency constraints that are added to the objective function $\left(\mathbf{v}_{c}\right)$ take into account the consistency with: higher elements in the hierarchy (top-down decomposition); lower elements in the hierarchy (bottom-up decomposition); and/or neighboring elements (non-hierarchic decomposition).

Relaxation of the consistency constraints is accomplished via:

1. Penalty functions, e.g., the quadratic norm of the inconsistency, ${ }^{22,52}$ quadratic norm of the inconsistency multiplied by a weight parameter ${ }^{41}$ and additional slack variables, ${ }^{58}$ inexact and exact penalty functions, ${ }^{31,32}$ amongst others.

2. Lagrangian relaxation, e.g., Lassiter et al. $(2005)^{47}$ and Kim et al. (2006). ${ }^{40}$

3. Augmented Lagrangian relaxation, e.g., Rodriguez et al. (2000), ${ }^{57}$ Blouin et al. $(2005)^{20}$ and Tosserams et al. $(2006-2010) \cdot{ }^{77-83}$ 


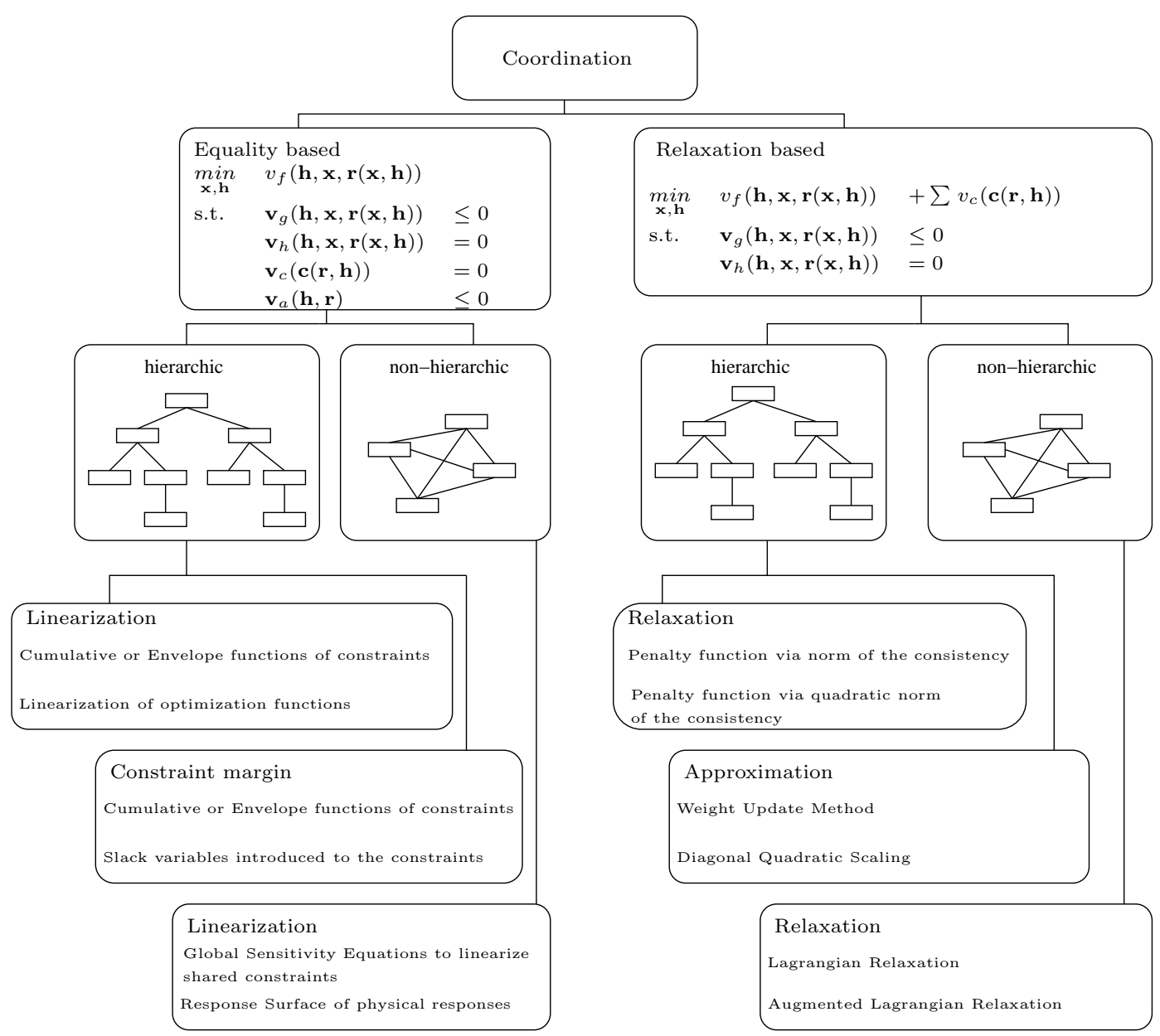

Figure 15. Overview of coordination approaches classified into two main stream approaches. The first (left): methods that coordinate individual elements where the decomposition is based on equality consistency constraints. The second (right): methods that coordinate individual elements where the decomposition is based on relaxed consistency constraints. Each main stream approach is subdivided into three classifications. The equality-based coordination into: linearization, approximation and non-hierarchic linearization methods. The relaxation-based coordination into: relaxation, approximation and non-hierarchic relaxation. Each bi-level optimization method discussed in this work falls into one of these categories.

Coordination of elements where consistency is relaxed involves evaluating new relaxation parameters and updating of data related to the coupling between elements. The relaxation parameters are evaluated and updated either via direct computation or they are approximated. Likewise, exchange of data related to the coupling between elements is computed directly or approximated. Common approaches to deal with approximating relaxation parameters and coupling data are:

1. a weight update method. ${ }^{53}$

2. a linearization method. ${ }^{38}$

3. diagonal quadratic scaling. ${ }^{35,48}$

In Figure 15 an overview of the main stream approaches to coordination of optimization problems with embedded hierarchy is presented. A distinction is made between coordination that is applied to elements that are decomposed via equality consistency constraints (left) and coordination that is applied to elements that are decomposed via relaxed consistency constraints (right). Furthermore, a distinction between hierarchic and non-hierarchic coordination is given. 


\section{Solution sequence}

Final step in applying a multi-level and/or multi-disciplinary optimization approach is to choose a solution method. Figure [16 shows the main components of a flowchart for multi-level and/or multi-disciplinary optimization. In literature a number of papers discuss steps to generalize these main components into a single (software) framework. ${ }^{5-8,26,29,34,51}$ Initial step is setting up the multi-level optimization problem. This is accomplished automated ${ }^{9,10}$ or manually via the steps discussed in the previous sections. Depending on the type of multi-level optimization approach chosen, additional information is required from neighboring elements. The most inner loop indicated via number 1 in Figure 16 represents the construction of e.g. optimum sensitivities. These are required to solve individual optimization problems located e.g. higher (using a top-down hierarchic decomposition) in the multi-level hierarchy.

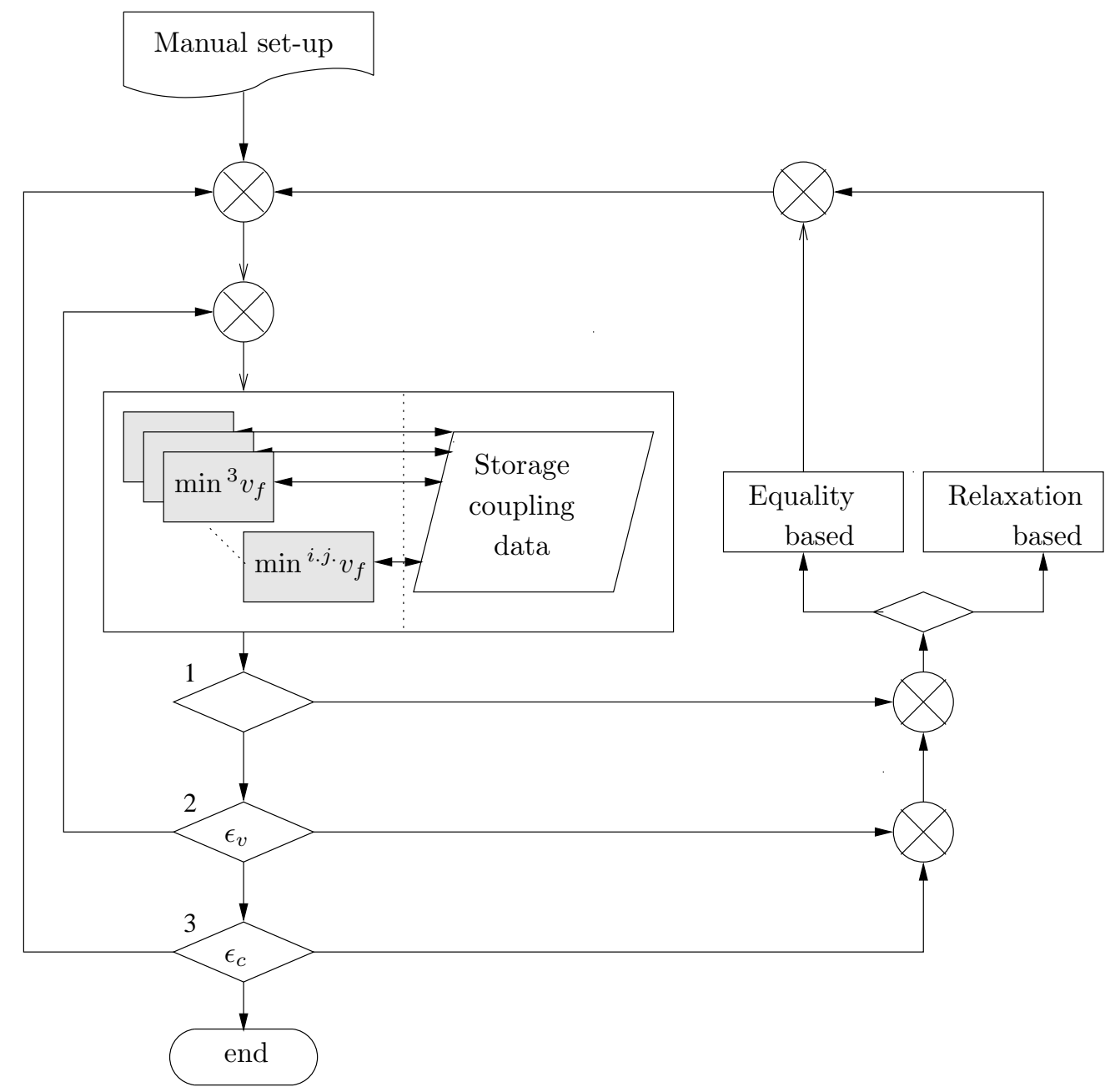

Figure 16. Flowchart showing the main components of a multi-level optimization process.

Once a hierarchy of individual coupled optimization problems has been defined these individual optimization problems can be solved separately. After an individual optimization problem is solved, coupling parameters $(\cdot . \mathbf{h}, \cdot . \mathbf{z})$ are stored separately for usage by neighboring elements to which the current element is coupled. Individual elements can be solved sequentially ${ }^{29}$ (Gauss-Seidel $\left.{ }^{18}\right)$, in parallel ${ }^{29}\left(\mathrm{Jacobi}^{18}\right)$ or via a combination. ${ }^{26}$ Choices mainly depend on the decomposition and coordination approach chosen or available infrastructure.

After the entire hierarchy is solved once, convergence of the individual objective functions is measured corresponding to loop 2 (or inner-loop ${ }^{82}$ ) in Figure 16 Via an error tolerance $\varepsilon_{v}$ the reduction in objective function value is determined. Depending on the outcome, control is given to the coordinator that is either equality based or relaxation based. Here decisions are taken to re-evaluate the hierarchy and/or to update 
approximations. In addition, a second convergence criterium can be evaluated via loop 3 (outer-loop ${ }^{82}$ ) measuring the size (or rate of decrease) of inconsistency $\varepsilon_{c}$. Depending on the outcome, e.g. relaxation parameters are updated. ${ }^{26}$

\section{Challenges for multi-level optimization}

Multi-level optimization and multi-disciplinary design optimization methods are practical when the amount of data that is exchanged between individual elements remains small and models are weakly coupled. Weak coupling in the sense that large errors in the solution of a neighboring element do not occur when mapping the data from one element onto a neighboring element. Furthermore, for equality-based and relaxation-based methods we observed ${ }^{26-29}$ that:

- Equality-based methods require that for each parameter that is send from one element to a neighboring element either sensitivity information is required or a response surface is constructed. Constructing accurate sensitivity information is not straightforward and can become a numerically expensive procedure on its own. Fitting a response surface through elements that output more then ten parameters to a single neighboring element (a small number if a detailed local finite element model communicates physical responses and/or shared design variables with a global finite element model) is challenging.

- Relaxation-based methods are known to exhibit poor numerical convergence characteristics. The linear convergence rate of these methods and the fact that intermediate designs during the multi-level optimization cannot be used makes these methods challenging to use in a competitive environment.

Techniques that seem promising with respect to convergence characteristics are developed by DeMiguel and Nogales (2008). ${ }^{32}$ The authors propose the use of Schur-Interior Point methods to coordinate the individual optimization problems. These methods exhibit super linear or even quadratic convergence characteristics as opposed to bi-level coordination methods that were discussed in this work. Similarly, Alexandrov et al. ${ }^{1-3}$ proposes the use of Null-space methods ${ }^{17,33}$ to coordinate the individual optimization problems. In addition, other approaches based on approximating search directions have been document in the literature. ${ }^{25} \mathrm{~A}$ drawback of such methods is that the same optimization technique should be used in each individual element. In this work each individual optimization problem from a hierarchy can be solved by different optimization techniques.

\section{Conclusion}

This paper identified four generic steps from which multi-level optimization methods are constructed. Physical coupling was illustrated via a coupling circle that shows the difference between top-down, bottomup and non-hierarchic decomposition. Furthermore, two distinct decomposition approaches were defined: strong (equality) consistency constraint formulations and weak (relaxed) consistency constraint formulations. How this coupling enters the optimization problem was shown via a problem matrix for which four distinct patterns can be identified. These patterns identify problem structure of optimization problems encountered in engineering. Coordination was introduced according to the decision making process. Decisions on which element of the hierarchy to solve first have an impact on algorithm accuracy and performance. Multi-level optimization and multi-disciplinary optimization techniques defined in the literature were shown as examples of equality-based or relaxation-based coordination approaches. Via a flowchart the solution process of multilevel and multi-disciplinary optimization approaches was presented. Finally, based on previous studies and results found in the literature we concluded that multi-level optimization and multi-disciplinary design optimization methods are practical when the amount of data that is exchanged between individual elements remains small and models are weakly coupled.

\section{Acknowledgments}

This research was part of the larger project Multiscale Methods in Computational Mechanics which was financially supported by the Dutch Technology Foundation - STW, the Netherlands Organisation for Scientific Research - NWO (DFG 5790) and the German Research Foundation - DFG (FOR 509). 


\section{References}

${ }^{1}$ N. Alexandrov and J.E. Dennis. Multilevel algorithms for nonlinear optimization. In J.B. Bogged and M. JohnGunzburger, editors, Optimal design and control; Proceedings of the Workshop on Optimal Design and Control, April 1994.

${ }^{2}$ N.M. Alexandrov. Multilevel methods for mdo. In N.M. Alexandrov and M.Y. Hussaini, editors, Multidisciplinary design optimization - state of the art. SIAM, Philadelphia, 1997.

${ }^{3}$ N.M. Alexandrov. A Trust-Region Framework for Managing Approximations in Constrained Optimization and MDO Problems. In ISSMO/NASA 1st Internet Conference on Approximations and Fast Reanalysis in Engineering Optimization, June 14-27, pages 1-14, 1998.

${ }^{4}$ N.M. Alexandrov and R.M. Lewis. Comparative properties of collaborative optimization and other approaches to mdo. Technical Report ICASE Report No. 99-24, Institute for Computer Applications in Science and Engineering, NASA Langley Research Center, Hampton, Virigina, July 1999.

${ }^{5}$ N.M. Alexandrov and R.M. Lewis. Algorithmic perspectives on problem formulations in mdo. In 8th AIAA/USAF/NASA/ISSMO Symposium on Multidisciplinary Analysis $\&$ Optimization, september 2000.

${ }^{6}$ N.M. Alexandrov and R.M. Lewis. Analytical and computational properties of distributed approaches to mdo. In 8 th AIAA/USAF/NASA/ISSMO Symposium on Multidisciplinary Analysis \& Optimization, september 2000.

${ }^{7}$ N.M. Alexandrov and R.M. Lewis. Dynamically reconfigurable approach to multidisciplinary problems. In 16 th AIAA Computational Fluid Dynamics Conference, Orlando, Florida, June 2003.

${ }^{8}$ N.M. Alexandrov and R.M. Lewis. Reconfigurability in mdo problem synthesis, part 2. In 10th AIAA/ISSMO Multidisciplinary Analysis and Optimization Conference, Albany, New York, August 2004.

${ }^{9}$ J. T. Allison, M. Kokkolaras, and P.Y. Papalambros. Optimal partitioning and coordination decisions in decompositionbased design optimization. Journal of Mechanical Design, 131, 2009.

${ }^{10}$ J.T. Allison and P.Y. Papalambros. Consistency constraint allocation in augmented lagrangian coordination. In Proceedings of the ASME 2008 International Design Engineering Technical Conferences and Computers and Information in Engineering Conference, IDETC/CIE, Brooklyn, New York, USA, August 2008.

${ }^{11}$ S.S. Altus, I.M. Kroo, and P.J. Gage. A genetic algorithm for scheduling and decomposition of multidisciplinary design problems. Transactions of the ASME.

${ }^{12}$ R.J. Balling and J. Sobieszczanski-Sobieski. An algorithm for solving the system-level problem in multilevel optimization. Structural Optimization, 9:168-177, 1995.

${ }^{13}$ R.J. Balling and J. Sobieszczanski-Sobieski. Optimization of coupled systems: A critical overview of approaches. American Institute of Aeronautics and Astronautics Journal, 34, 1996.

${ }^{14}$ J.-F. M. Barthelemy and J. Sobieszczanski-Sobieski. Optimum sensitivity derivatives of objective functions in nonlinear programming. Technical notes, AIAA, 1983.

${ }^{15}$ J.-F.M. Barthelemy. Engineering design applications of multilevel optimization methods. In C.A. Brebbia and S. Hernandez, editors, Computer Aided Optimum Design of Structures: Applications. Springer-Verlag, 1989.

${ }^{16} \mathrm{M}$. Beers and G.N. Vanderplaats. A linearization method for multilevel optimization. In Proceedings of the international conference on numerical methods in engineering: Theory and applications/SWANSEA. Martinus Nijhoff Publishers, July 1987.

${ }^{17}$ Michele Benzi, Gene H. Golub, and Lörg Liesen. Numerical solution of saddle point problems. Acta Numerica, pages 1-137, 2005.

${ }^{18}$ D.P. Bertsekas. Nonlinear Programming. Athena Scientific, Belmont, Massachusetts, 1995.

${ }^{19}$ C.L. Bloebaum. Coupling strenght-based system reduction for complex engineering design. Structural Optimization, 10:113-121, 1995.

${ }^{20}$ V.Y. Blouin, J.B. Lassiter, M.M. Wiecek, and G.M. Fadel. Augmented lagrangian coordination for decomposed design problems. In sixth World Congress of Structural and Multidisciplinary Optimization, 2005.

${ }^{21}$ V.Y. Blouin, J.D. Summers, G.M. Fadel, and J. Gu. Intrinsic analysis of decomposition and coordination strategies for complex design problems. In 10th AIAA/ISSMO Multidisciplinary Analysis and Optimization Conference 30 August - 1 September 2004, New York, 2004.

${ }^{22}$ R.D. Braun and I. Kroo. Development and application of the collaborative optimization architecture in a multidisciplinary design environment. In N.M. Alexandrov and M.Y. Hussaini, editors, Multidisciplinary Design Optimization, State of the Art. Philadelphia: SIAM, 1997.

${ }^{23} \mathrm{~L}$. Chen and S. Li. Analysis of decomposability and complexity for design problems in the context of decomposition. Journal of Mechanical Design, 127, 2005.

${ }^{24}$ S. Chen, F. Zhang, and M. Khalid. Evaluation of three decomposition mdo algorithms. In Published by the International Council of the Aeronautical Sciences ICAS 2002, 2002.

${ }^{25}$ A.J. Conejo, F.J. Nogales, and F.J. Prieto. A decomposition procedure based on approximate newton directions. Mathematical Programming, 93(3):1436-4646, 2002.

${ }^{26}$ A.J. de Wit. A unified approach towards decomposition and coordination for multi-level optimization. PhD thesis, Delft University of Technology, 2009.

${ }^{27}$ A.J. de Wit and F. van Keulen. Numerical Comparison of Multi-Level Optimization Techniques. In 3rd AIAA Multidisciplinary Design Optimization Specialist Conference, Waikiki in Honolulu, Hawaii, 23-26 April, 2007.

${ }^{28}$ A.J. de Wit and F. van Keulen. Framework for multilevel optimization. In CJK-OSM5, 5th China-Japan-Korea Joint Symposium on Optimization of Structural and Mechanical Systems, Jeju Island, South Korea, 16-19 April, 2008.

${ }^{29}$ A.J. de Wit and F. van Keulen. Framework for multi-level optimization of complex systems. In E. Ramm and R. de Borst, editors, Lecture Notes in Applied and Computational Mechanics (LNACM). Springer, to be published, 2010.

${ }^{30} \mathrm{~V}$. DeMiguel and W. Murray. An analysis of collaborative optimization methods. In proceedings of the 8th AIAA/USAF/NASA/ISSMO Symposium on Multidisciplinary Analysis and Optimization, 2000. 
${ }^{31} \mathrm{~V}$. DeMiguel and W. Murray. A local convergence analysis of bilevel decomposition algorithms. Optimization and Engineering, 7:99-133, 2006.

${ }^{32}$ V. DeMiguel and F.J. Nogales. On Decomposition Methods for a Class of Partially Separable Nonlinear Programs. Mathematics of Operations Research, pages 119-139, 2008.

${ }^{33}$ J. Dennis, M. El-Alem, and M.C. Maciel. A Global Convergence Theory for General Trust-Region-Based Algorithms for Equality Constrained Optimization. Technical report tr92-28, Department of Computational and Applied Mathematics, Rice university, Houston, Texas, 1997.

${ }^{34}$ L.F.P. Etman, M. Kokkolaras, A.T Hofkamp, P.Y. Papalambros, and J.E. Rooda. Coordination specification in distributed optimal design of multilevel systems using the $\chi$ language. Structural and Multidisciplinary Optimization, 29:198-212, 2005.

${ }^{35}$ A.A. Groenwold, L.F.P. Etman, S. Kok, D.W. Wood, and S. Tosserams. An augmented lagrangian approach to non-convex sao using diagonal quadratic approximations. Structural and Multidisciplinary Optimization, 38:415-421, 2009.

${ }^{36}$ R.T. Haftka and L.T. Watson. Multidisciplinary Design Optimization with Quasiseparable Subsystems. Optimization and Engineering, 6:9-20, 2005.

${ }^{37}$ R.T. Haftka and L.T. Watson. Decomposition theory for multidisciplinary design optimization problems with mixed integer quasiseparable subsystems. Optimization and Engineering, 7:135-149, 2006.

${ }^{38} \mathrm{~J}$. Han and P. Papalambros. A sequential linear programming coordination algorithm for analytical target cascading. In Proceedings of the ASME 2007 International Design Engineering Technical Conferences and Computers and Information in Engineering Conference, IDETC/CIE, Las Vegas, Nevada, USA, September 2007.

${ }^{39}$ H.M. Kim. Target Cascading in Optimal System Design. PhD thesis, The University of Michigan, 2001.

${ }^{40}$ H.M. Kim, W. Chen, and M.M. Wiecek. Lagrangian coordination for enhancing the convergence of analytical target cascading. American Institute of Aeronautics and Astronautics Journal, 44(10):2197-2207, 2006.

${ }^{41}$ H.M. Kim, N.F. Michelena, P.Y. Papalambros, and T. Jiang. Target cascading in optimal system design. Journal of Mechanical Design, 125:474-480, September 2003.

${ }^{42}$ U. Kirsch. Two-level optimization of prestressed structures. Engineering Structures, 19(4):309-317, 1997. 1979.

${ }^{43}$ U. Kirsch and F. Moses. Decomposition in optimum structural design. Journal of the structural division, 105:85-100,

${ }^{44}$ S. Kodiyalam. Evaluation of methods for multidisciplinary design optimization (mdo), phase i. Technical Report CR1998-208716, NASA, September 1998.

${ }^{45} \mathrm{~S}$. Kodiyalam and J. Sobieszczanski-Sobieski. Multidisciplinary design optimization - some formal methods, framework requirements, and application to vehicle design. International Journal of Vehicle Design, 25:3-22, 2001.

${ }^{46} \mathrm{~S}$. Kodiyalam and C. Yuan. Evaluation of methods for multidisciplinary design optimization (mdo), part ii. Technical Report CR-2000-210313, NASA, November 2000.

${ }^{47}$ J.B. Lassiter, M.M. Wiecek, and K.R. Andrighetti. Lagrangian coordination and analytical target cascading: Solving atc-decomposed problems with lagrangian duality. Optimization and Engineering, 6:361-381, 2005.

${ }^{48}$ Y. Li, Z. Lu, and J. Michalek. Diagonal Quadratic Approximation for Parallelization of Analytical Target Cascading. ASME Journal of Mechanical Design, 130(5), 2008.

${ }^{49}$ J.G. Lin. Analysis and enhancement of collaborative optimization for multidisciplinary design. American Institute of Aeronautics and Astronautics Journal, 42, 2004.

${ }^{50}$ B. Liu, R.T. Haftka, and L.T. Watson. Global-local structural optimization using response surfaces of local optimization margins. Structural and Multidisciplinary Optimization, 27:352-359, 2004.

${ }^{51}$ J.R.R.A. Martins, C. Marriage, and N. Tedford. pymdo: An object-oriented framework for multidisciplinary design optimization. ACM Transactions on Mathematical Software, 36, 2009.

${ }^{52}$ C.D. McAllister, T.W. Simpson, K. Hacker, K. Lewis, and A. Messac. Integrating linear physical programming within collaborative optimization for multiobjective multidisciplinary design optimization. Structural and Multidisciplinary Optimization, 29:178-189, 2005.

${ }^{53}$ J.J. Michalek and P.Y. Papalambros. An efficient weighting update method to achieve acceptable consistency deviation in analytical target cascading. Journal of Mechanical Design, 127:206-214, 2005.

${ }^{54}$ J. Pan and A.R. Diaz. Some results in optimization of non-hierarchic systems. advances in design automation - Volume Two Design Optimization, DE-Vol. 19-2:15-20, 1989.

${ }^{55}$ J.E. Renaud and G.A. Gabriele. Improved Coordination in Nonhierarchic System Optimization. American Institute of Aeronautics and Astronautics Journal, 31:2367-2373, December 1993.

${ }^{56}$ J.E. Renaud and G.A. Gabriele. Approximation in Nonhierarchic System Optimization. American Institute of Aeronautics and Astronautics Journal, 32:198-205, January 1994.

${ }^{57}$ J.F. Rodríguez, J.E. Renaud, B.A. Wujek, and R.V. Tappeta. Trust region model management in multidisciplinary design optimization. Journal of Computational and Applied Mathematics, 124:139-154, 2000.

${ }^{58}$ B.D. Roth and I.M. Kroo. Enhanced collaborative optimization: A decomposition-based method for multidisciplinary design. In Proceedings of the ASME 2008 International Design Engineering Technical Conferences and Computers and Information in Engineering Conference IDETC/CIE,Brooklyn, New York, USA, August 2008.

${ }^{59}$ J.F. Schutte, R.T. Haftka, and L.T. Watson. Decomposition and two-level optimization of structures with discrete sizing variables. American Institute of Aeronautics and Astronautics Journal, 2004.

${ }^{60}$ R.S. Sellar, M.A. Stelmack, S.M. Batill, and J.E. Renaud. Response surface approximations for discipline coordination in multidisciplinary design optimization. AIAA/ASME/ASCE/AHS/ASC 37th Structures, Structural Dynamics and Materials Conference, Salt Lake City, Utah, (AIAA-96-1383):583-593, April 1996.

${ }^{61}$ J. Shankar, C.J. Ribbens, R.T. Haftka, and L.T. Watson. Computational study of a nonhierarchical decomposition algorithm. Computational Optimization and Applications, 2:273-293, 1993.

${ }^{62}$ M.-K. Shin and G.-J. Park. Multidisciplinary design optimization based on independent subspaces. International Journal for Numerical Methods in Engineering, 64:599-617, 2005. 
${ }^{63}$ J.A. Shupe, F. Mistree, and J. Sobieszanski-Sobieski. Compromise: An effective approach for the hierarchical design of structural systems. Computers and Structures, 26:1027-1037, 1987.

${ }^{64} \mathrm{~J}$. Sobieszczanski-Sobieski. A step from hierarchic to non-hierarchic systems. In 2nd NASA Air Force symposium on recent advances in multidisciplinary analysis and optimization, september 1988.

${ }^{65} \mathrm{~J}$. Sobieszczanski-Sobieski. Sensitivity analysis and multidisciplinary optimization for aircraft design: Recent advances and results. Journal of Aircraft, 27(12):993-1001, 1990.

${ }^{66}$ J. Sobieszczanski-Sobieski. Sensitivity of complex, internally coupled systems. AIAA Journal, 28(1):153-160, 1990.

${ }^{67} \mathrm{~J}$. Sobieszczanski-Sobieski. Sensitivity of Control-Augmented Structure Obtained by a System Decomposition Method. American Institute of Aeronautics and Astronautics Journal, 29:264-270, 1991.

${ }^{68} \mathrm{~J}$. Sobieszczanski-Sobieski. A technique for locating function roots and satisfying equality constraints in optimization. Structural Optimization, 4:241-243, 1992.

${ }^{69} \mathrm{~J}$. Sobieszczanski-Sobieski. Two alternative ways for solving the coordination problem in multilevel optimization. Structural Optimization, 6:205-215, 1993.

${ }^{70}$ J. Sobieszczanski-Sobieski, J.S. Agte, and R.R. Sandusky JR. Bi-level integrated system synthesis (bliss). In AIAA-984916, 1998.

${ }^{71}$ J. Sobieszczanski-Sobieski, J.S. Agte, and R.R. Sandusky JR. Bi-level integrated system synthesis (bliss). American Institute of Aeronautics and Astronautics Journal, 38(1):164-172, 2000.

${ }^{72}$ J. Sobieszczanski-Sobieski, T.D. Altus, M. Phillips, and R. Sandusky. Bilevel integrated system synthesis for concurrent and distributed processing. American Institute of Aeronautics and Astronautics Journal, 41(10):1996-2003, October 2003.

${ }^{73}$ J. Sobieszczanski-Sobieski, J. F. Barthelemy, and K.M. Riley. Sensitivity of optimum solutions of problem parameters. American Institute of Aeronautics and Astronautics Journal, 20:1291-1299, 1982.

${ }^{74}$ J. Sobieszczanski-Sobieski and R.T. Haftka. Multidisciplinary aerospace design optimization: survey of recent developments. Structural Optimization, 14:1-23, 1997.

${ }^{75}$ J. Sobieszczanski-Sobieski, B.B. James, and A.R. Dovi. Structural optimization by multilevel decomposition. American Institute of Aeronautics and Astronautics Journal, 23:124-142, 1985.

${ }^{76}$ J. Sobieszczanski-Sobieski, B.B. James, and M.F. Riley. Structural sizing by generalized, multilevel optimization. American Institute of Aeronautics and Astronautics Journal, 25:139-145, 1987.

${ }^{77} \mathrm{~S}$. Tosserams. Distributed optimization for systems design. PhD thesis, Eindhoven University of Technology, 2008.

${ }^{78}$ S. Tosserams, L.F.P. Etman, P.Y. Papalambros, and J.E. Rooda. An augmented lagrangian relaxation for analytical target cascading using the alternating direction method of multipliers. Structural and Multidisciplinary optimization, 31:176189, 2006.

${ }^{79}$ S. Tosserams, L.F.P. Etman, and J.E. Rooda. An augmented lagrangian decomposition method for quasi-separable problems in mdo. Structural and Multidisciplinary Optimization, 34(3):211-227, 2007.

${ }^{80}$ S. Tosserams, L.F.P. Etman, and J.E. Rooda. Augmented lagrangian coordination for distributed optimal design in MDO. International Journal for Numerical Methods in Engineering, 73:1885-1910, 2008.

${ }^{81}$ S. Tosserams, L.F.P. Etman, and J.E. Rooda. Performance evaluation of augmented lagrangian coordination for distributed multidisciplinary design optimization. In Proceedings of the 4 th AIAA Multidisciplinary Design Optimization Specialist Conference, Schaumburg, IL, United States, 1-17, 2008.

${ }^{82}$ S. Tosserams, L.F.P. Etman, and J.E. Rooda. A classification of methods for distributed system optimization based on formulation structure. Structural and Multidisciplinary Optimization, 39:503-., 2009.

${ }^{83}$ S. Tosserams, A.T. Hofkamp, L.F.P. Etman, and J.E. Rooda. A micro-accelerometer mdo benchmark problem. Structural Multidisciplinary Optimization, Accepted, to appear, 2009.

${ }^{84}$ G.N. Vanderplaats, Y.J. Yang, and D.S. Kim. Sequential linearization method for multilevel optimization, old. American Institute of Aeronautics and Astronautics Journal, 28:290-295, 1990.

${ }^{85}$ T.C. Wagner. A general decomposition methodology for optimal system design. PhD thesis, The University of Michigan, 1993.

${ }^{86}$ P.M. Zadeh, V.V. Toropov, and A.S. Wood. Metamodel-based collaborative optimization framework. Structural and Multidisciplinary Optimization, 38:103-115, 2009. 